\title{
A New Parametric Lifetime Distribution with Modified Chi-square Type Test for Right Censored Validation, Characterizations and Different Estimation Methods
}

\author{
Haitham M. Yousof ${ }^{1 *}$, Khaoula Aidi², G.G. Hamedani ${ }^{3}$ and Mohamed Ibrahim ${ }^{4}$ \\ * Corresponding Author
}

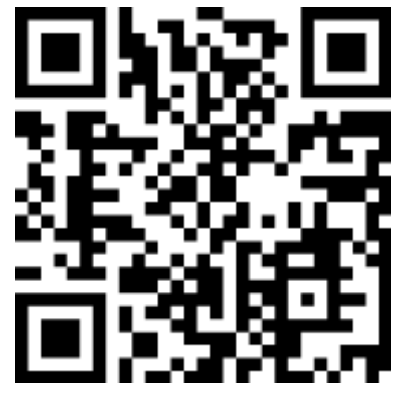

1. Department of Statistics, Mathematics and Insurance, Benha University, Benha, Egypt. haitham.yousof@fcom.bu.edu.eg

2. Laboratory of probability and statistics LaPS, University Badji Mokhtar, Annaba, Algeria. khaoula.aidi@yahoo.fr

3. Department of Mathematical and Statistical Sciences, Marquette University, USA. gholamhoss.hamedani@marquette.edu

4. Department of Applied, Mathematical and Actuarial Statistics, Faculty of Commerce, Damietta University, Damietta,

Egypt. mohamed_ibrahim@du.edu.eg

Abstract: A new three-parameter extension of the generalized Nadarajah-Haghighi model is introduced and studied. Some of its statistical properties are derived. Characterization results are presented. The failure rate can be "increasing", "decreasing", "bathtub", "upside-down", "upside-down-constant", "increasing-constant" or "constant". Different non-Bayesian estimation methods under uncensored scheme are considered. Numerical simulations are performed for comparing the estimation methods using different sample sizes. The censored Barzilai-Borwein algorithm is employed via a simulation study. Using the approach of the Bagdonavicius-Nikulin chi-square goodness-of-fit test for validation under the right censored data, we propose a modified chi-square goodness-of-fit test for the new model. Based on the maximum likelihood estimators on initial data, the modified Bagdonavicius-Nikulin chi-square goodness-of-fit test recovers the loss in information. The modified Bagdonavicius-Nikulin test for validation under the right censored data is applied to four real and right censored data sets. The new model is compared with many other competitive models by means of a real data set.

Keywords: Bagdonavicius-Nikulin test; Nadarajah Haghighi Model; Non-Bayesian; Characterizations; Morgenstern Family; Clayton Copula; Moments; Numerical Analysis; Simulation; Censored Barzilai-Borwein.

Mathematical Subject Classification: 62N01; 62N02; 62E10.

\section{Introduction}

Lemonte (2013) proposed a new three-parameter model called the generalized Nadarajah-Haghighi (GNH). He proved that the hazard rate function (HRF) of his new model can be "increasing", "decreasing", "bathtub (U-HRF) or "upsidedown". The cumulative distribution function (CDF) of the GNH model is given by

$$
\boldsymbol{\Pi}_{b, \theta, \beta}(z)=\left.\left\{1-\exp \left[1-(z \beta+1)^{\theta}\right]\right\}^{b}\right|_{(z \geq 0)}
$$

where $b>0$ and $\theta>0$ are shape parameters and $\beta>0$ is scale parameter. Clearly, when $b=\theta=1$, we have the standard exponential (E) model. For $\theta=1$, the GNH model reduces to the generalized exponential (GE) model. For $b=1$, the GNH model reduces to the Nadarajah and Haghighi (NH) model (see Nadarajah and Haghighi (2011)). Recently, many authors defined and studied many probability distributions. In this work and after proposing a new model called the Burr X generalized Nadarajah-Haghighi (BXGNH), a special Section is devoted to the characterizations of the BXGNH distribution based on a simple relationship between two truncated moments; in terms of the HRF and based on the conditional expectation of a function of the random variable (RV). Mathematical and statistical properties are also studied along with a numerical analysis for certain measures.

Different non-Bayesian estimation methods under uncensored scheme are considered for estimating such as the maximum likelihood estimation (MLE) method, Anderson Darling estimation (ADE) method, ordinary least square estimation (OLSE) method, Cramér-von-Mises estimation (CVME) method, weighted least square estimation 
(WLSE) method, left tail ADE (LTADE) method and right tail ADE (RTADE) method. For comparing non-Bayesian estimation methods, numerical simulations are performed using different sample sizes. For comparing the competitive distributions under the uncensored MLE method, an application is presented. The censored Barzilai-Borwein (BB) algorithm is employed via a simulation study for assessing the performance of the estimators with different sample sizes as the sample size tends to $+\infty$. Using the approach of the Bagdonavicius-Nikulin chi-square goodness-of-fit test for validation under the right censored data, we propose a modified chi-square goodness-of-fit test for the BXGNH model. The modified goodness-of-fit statistic test is applied for four right censored real data set. Based on the maximum likelihood estimators (MLEs) on initial data, the modified Bagdonavicius-Nikulin chi-square goodness-offit test recovers the loss in information. All elements of the modified Bagdonavicius-Nikulin chi-square goodness-offit test are explicitly derived and given.

According to Yousof et al., (2017), the CDF and the probability density function (PDF) of the BX-G family of distributions can be expressed as

$$
F_{a, \underline{\xi}}(z)=\left\{1-\exp \left[-\boldsymbol{O}_{\underline{\xi}}^{2}(z)\right]\right\}^{a}
$$

where $\boldsymbol{\mathcal { O }}_{\underline{\xi}}(z)=\boldsymbol{\Pi}_{\underline{\xi}}(z) / \overline{\boldsymbol{\Pi}}_{\underline{\xi}}(z)$. The corresponding PDF can be written as

$$
f_{a, \underline{\xi}}(z)=2 a \frac{\pi_{\underline{\xi}}(z) \boldsymbol{\Pi}_{\underline{\xi}}(z)}{\overline{\boldsymbol{\Pi}}_{\underline{\xi}}(z)^{3} \exp \left[\boldsymbol{\mathcal { O }}_{\underline{\xi}}^{2}(z)\right]}\left\{1-\exp \left[-\boldsymbol{\mathcal { O }}_{\underline{\xi}}^{2}(z)\right]\right\}^{a-1},
$$

where $a>0$ is the shape parameter, $\pi_{(\underline{\Phi})}(z)$ and $\boldsymbol{\Pi}_{(\underline{\Phi})}(z)$ denote the PDF and CDF of the baseline model with parameter vector $\underline{\xi}, \overline{\boldsymbol{\Pi}}_{\underline{\xi}}(z)=1-\boldsymbol{\Pi}_{\underline{\xi}}(z)$ and $\pi_{\underline{\xi}}(z)=d \boldsymbol{\Pi}_{\underline{\xi}}(z) / d z$. To this end, we use $\boldsymbol{\Pi}_{b, \theta, \beta}(z)$ and equation (1) to obtain the four-parameter BXGNH CDF as

$$
\left.F_{\underline{\Phi}}(z)\right|_{(z \geq 0)}=\left(1-\exp \left\{-\left[\left(1-\boldsymbol{O}_{z ; \theta, \beta}\right)^{-b}-1\right]^{-2}\right\}\right)^{a},
$$

where $\underline{\boldsymbol{\Phi}}=(a, b, \theta, \beta), \boldsymbol{\mathcal { O }}_{z ; \theta, \beta}=\exp \left[1-(z \beta+1)^{\theta}\right]$. The corresponding PDF can be expressed as

$$
\left.f_{\underline{\Phi}}(z)\right|_{(z \geq 0)}=2 a b \theta \beta \frac{(z \beta+1)^{\theta-1} \exp \left\{-\left[\left(1-\boldsymbol{\mathcal { O }}_{z ; \theta, \beta}\right)^{-b}-1\right]^{-2}\right\}}{\boldsymbol{\mathcal { O }}_{z ; \theta, \beta}\left(1-\boldsymbol{\mathcal { O }}_{z ; \theta, \beta}\right)^{b+1}\left(1-\exp \left\{-\left[\left(1-\boldsymbol{\mathcal { O }}_{z ; \theta, \beta}\right)^{-b}-1\right]^{-2}\right\}\right)^{1-a}}
$$

The reliability function (RF), HRF, reversed hazard rate function (RHRF) and cumulative hazard rate function (CHRF) of $Z$ can be derived with the well-known relationships. Following Yousof et al., (2017), many authors presented many new useful life-time models such as Khalil et al., (2019) Burr X exponentiated Weibull model and Mansour et al., (2020d) for Burr X Weibull model. For $a=1$, the BXGNH distribution reduces to the BXNH model. For $a=\theta=1$, the BXGNH distribution reduces to the Rayleigh generalized exponential (RGE) model. For $\theta=1$, the BXGNH distribution reduces to the BX generalized exponential (BXGE) model. For $b=a=1$, the BXGNH distribution reduces to the Rayleigh $\mathrm{NH}(\mathrm{RNH})$ model. For $b=a=\theta=1$, the BXGNH distribution reduces to the Rayleigh exponential (RE) model. For $b=\theta=1$, the BXGNH distribution reduces to the BX exponential (BXE) model. For examining the fallibility of the BXGNH distribution we preset Figure 1 (for examining the fallibility of the PDF of the BXGNH distribution) and Figure 2 (for examining the fallibility of the HRF of the BXGNH distribution).

Figure 1 shows that the new PDF can be "unimodal with heavy tail ", the "symmetric", right skewed with heavy tail and no peak" and "right skewed with heavy tail and one peak". Figure 2 shows that the HRF may be "monotonically increasing" $(a=1, b=1, \theta=1, \beta=1)$, "monotonically decreasing" $(a=0.5, b=1.25, \theta=0.1, \beta=0.5)$, "upsidedown" ( $a=0.85, b=1, \theta=0.01, \beta=0.35)$, "upside-down-constant" $(a=0.4, b=3, \theta=0.2, \beta=0.2)$, "bathtub $(\mathbf{U}$-HRF)" $\quad(a=0.125, b=0.95, \theta=1.55, \beta=0.25)$, "increasing-constant" $(a=0.8, b=0.95, \theta=0.22, \beta=$ 0.11 ) or "constant" ( $a=0.45, b=3, \theta=0.21, \beta=0.21)$ or "reversed J HRF" $(a=0.8, b=95, \theta=0.22, \beta=$ $0.11)$. 

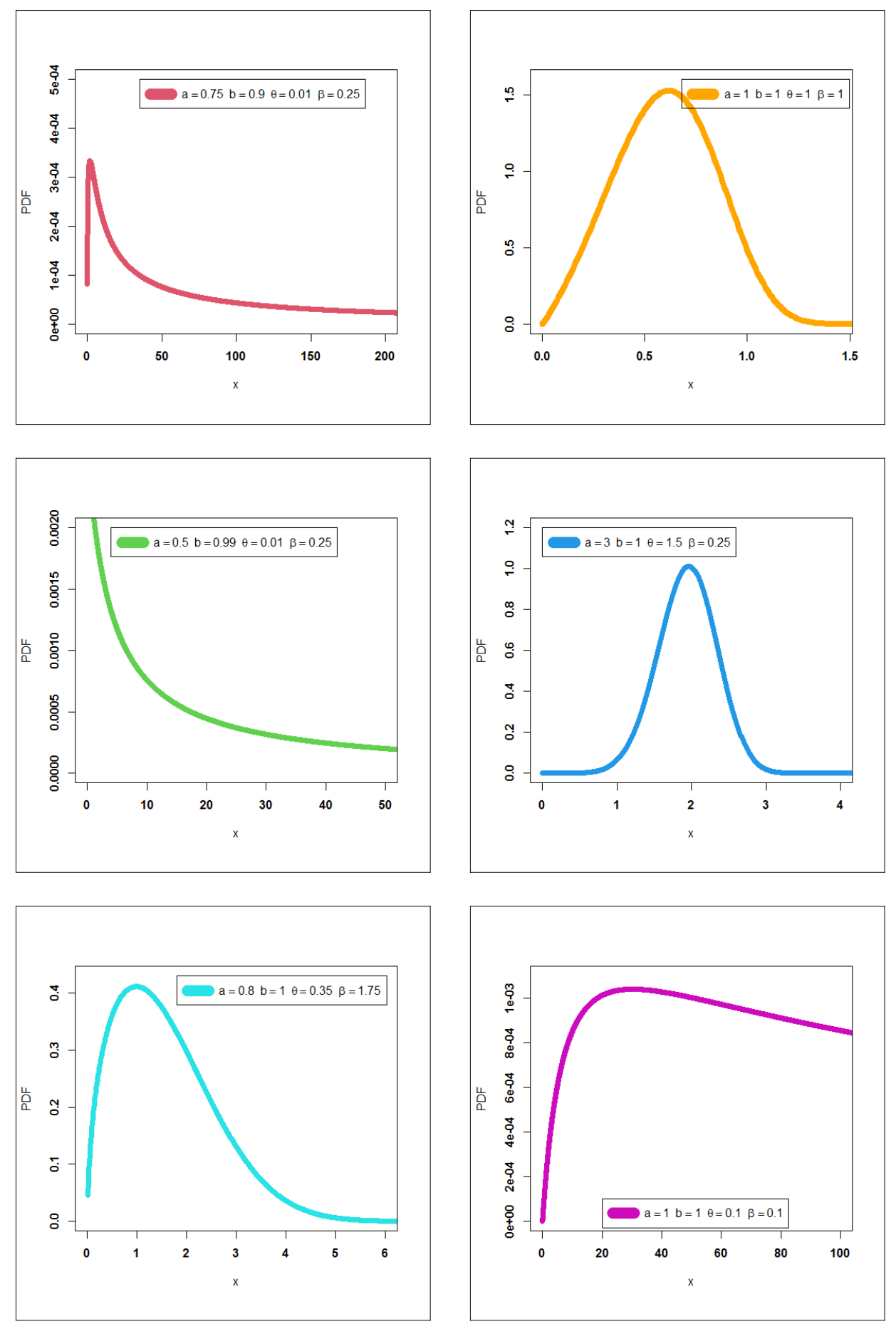

Figure 1: Plots of the BXGNH PDF for selected parameter values. 

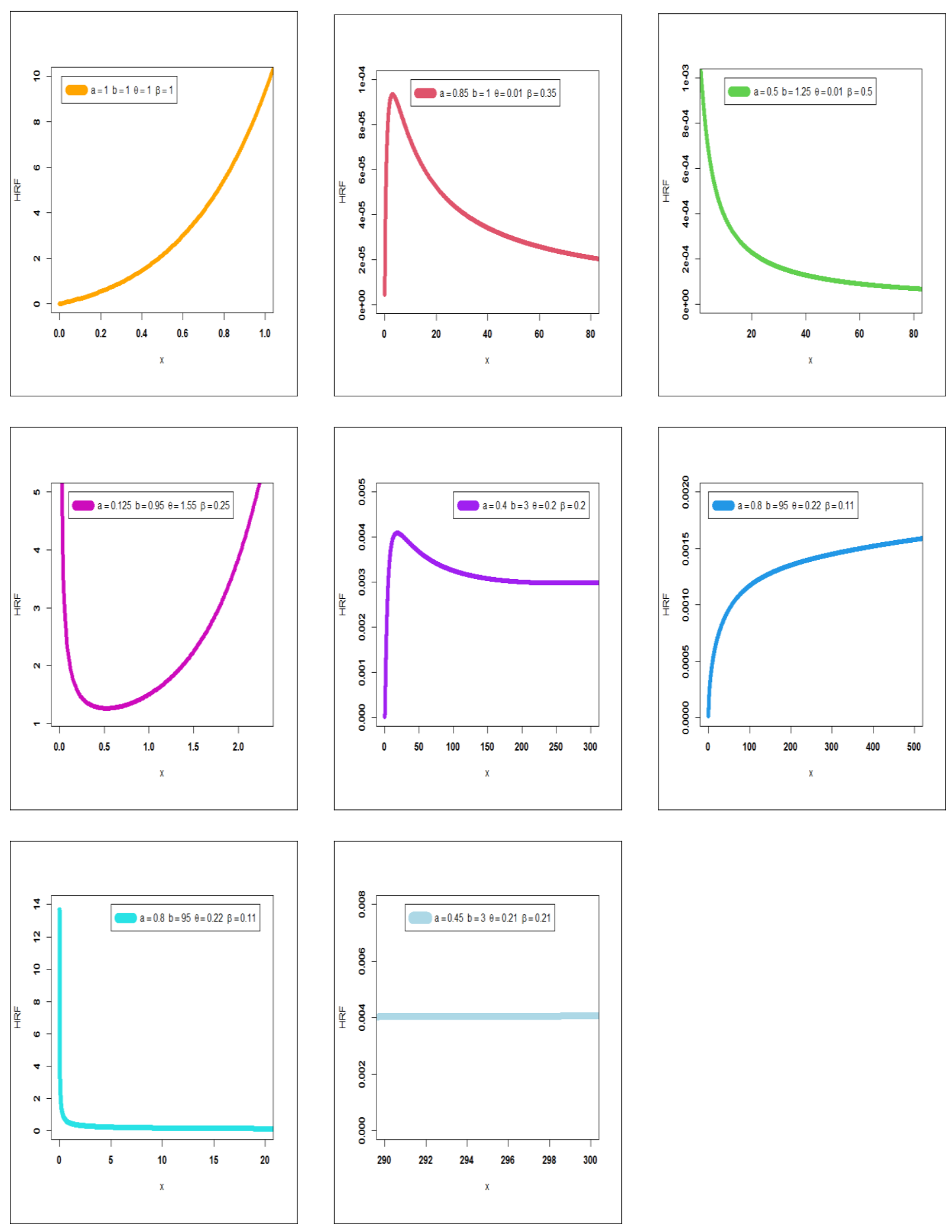

Figure 2: Plots of the BXGNH HRF for selected parameter values.

The statistical literature review contains many useful Nadarajah-Haghighi extensions such as see Nascimento et al., (2019), Elsayed and Yousof (2019), Alizadeh et al., (2018) and Ibrahim (2020) for more details. 


\section{Characterization results}

This section is devoted to the characterizations of the BXGNH distribution in the following directions:

(i) Based on a simple relationship between two truncated moments;

(ii) In terms of the HRF;

(iii) Based on the conditional expectation of a function of the RV.

It should be mentioned that for the characterization $(i)$ the CDF is not required to have a closed form. We present our characterizations $(\boldsymbol{i})-(\boldsymbol{i} i \boldsymbol{i})$ in three subsections.

\subsection{Characterizations based on two truncated moments}

In this subsection we present characterizations of the BXGNH distribution in terms of a simple relationship between two truncated moments. The first characterization result employs a theorem due to Glänzel (1987), see Theorem 2.1.1 below. Note that the result holds also when the interval $H$ is not closed. Moreover, as mentioned above, it could be also applied when the $\mathrm{CDF} F_{\underline{\Phi}}(z)$ does not have a closed form. As shown in Glänzel (1990), this characterization is stable in the sense of weak convergence.

\section{Theorem 2.1.1.}

Let $(\Omega, F, P)$ be a given probability space and let $H=[d, e]$ be an interval for some $d<e \quad(d=-\infty, e=$ $+\infty$ might as well be allowed). Let $z: \Omega \rightarrow H$ be a continuous RV with the distribution function $F_{\Phi}(z)$ and let $h_{1}$ and $h_{2}$ be two real functions defined on $H$ such that

$$
\boldsymbol{E}\left[h_{2}(Z) \mid Z \geq z\right]=\left.\boldsymbol{E}\left[h_{1}(Z) \mid Z \geq z\right] \tau(z)\right|_{(z \in H)},
$$

is defined with some real function $\tau$. Assume that $h_{1}, h_{2} \in C^{1}(H), \tau \in C^{2}(H)$ and $F$ is twice continuously differentiable and strictly monotone function on the set $H$. Finally, assume that the equation $\tau h_{1}=h_{2}$ has no real solution in the interior of $H$. Then $F$ is uniquely determined by the functions $h_{1}, h_{2}$ and $\tau$, particularly

$$
F_{\underline{\Phi}}(z)=\int_{a}^{z} C\left|\frac{\tau^{\prime}(u)}{\tau(u) h_{1}(u)-h_{2}(u)}\right| \exp [-s(u)] d u,
$$

where the function $s$ is a solution of the differential equation $s^{\prime}=\frac{\tau^{\prime} h_{1}}{\tau h_{1}-h_{2}}$ and $C$ is the normalization constant, such that $\int_{H} d F_{\underline{\Phi}}(z)=1$.

\section{Remark 2.1.1.}

The goal in Theorem 2.1.1 is to have $\tau(z)$ as simple as possible.

\section{Proposition 2.1.1.}

Let $Z: \Omega \rightarrow(0,+\infty)$ be a continuous RV and let

$$
h_{1}(z)=\frac{\left[1-\left(1-\boldsymbol{\mathcal { O }}_{z ; \theta, \beta}\right)^{b}\right]^{3} \exp \left\{\left[\left(1-\boldsymbol{\mathcal { O }}_{z ; \theta, \beta}\right)^{-b}-1\right]^{-2}\right\}}{\left(1-\boldsymbol{\mathcal { O }}_{z ; \theta, \beta}\right)^{2 b-1}\left(1-\exp \left\{-\left[\left(1-\boldsymbol{\mathcal { O }}_{z ; \theta, \beta}\right)^{-b}-1\right]^{-2}\right\}\right)}
$$

and $h_{2}(z)=\left.h_{1}(z) \boldsymbol{O}_{z ; \theta, \beta}^{b}\right|_{(z>0)}$. The RV $z$ has PDF (4) if and only if the function $\tau$ defined in Theorem 2.1 .1 has the form $\tau(z)=\left.\frac{1}{2} \boldsymbol{O}_{z ; \theta, \beta}\right|_{(z>0)}$.

Proof. Let $Z$ be a RV with PDF (4), then

$$
\left(1-F_{\underline{\Phi}}(z)\right) \boldsymbol{E}\left[h_{1}(Z) \mid Z \geq z\right]=\left.2 a b \boldsymbol{⿰}_{z ; \theta, \beta}\right|_{(z>0)},
$$

and

$$
\left(1-F_{\underline{\Phi}}(z)\right) \boldsymbol{E}\left[h_{2}(Z) \mid Z \geq z\right]=\left.a b \exp \left[2-2(z \beta+1)^{\theta}\right]\right|_{(z>0)},
$$

and finally

Conversely, if $\tau$ is given as above, then

$$
\tau(z) h_{1}(z)-h_{2}(z)=-\frac{1}{2} h_{1}(z) \boldsymbol{O}_{z ; \theta, \beta}<\left.0\right|_{(z>0)} .
$$

$$
s^{\prime}(z)=\frac{\tau^{\prime}(z) h_{1}(z)}{\tau(z) h_{1}(z)-h_{2}(z)}=\left.\beta \theta(z \beta+1)^{\theta-1}\right|_{(z>0)},
$$


and hence $s(z)=(z \beta+1)^{\theta}-1, z>0$. Now, in view of Theorem 2.1.1, $Z$ has density (4).

\section{Corollary 2.1.1.}

Let $Z: \Omega \rightarrow(0,+\infty)$ be a continuous RV and let $h_{1}(z)$ be as in Proposition 2.1.1. The PDF of $Z$ is $(4)$ if and only if there exist functions $h_{2}$ and $\tau$ defined in Theorem 2.1.1 satisfying the differential equation

\section{Corollary 2.1.2.}

$$
\frac{\tau^{\prime}(z) h_{1}(z)}{\tau(z) h_{1}(z)-h_{2}(z)}=\beta \theta(z \beta+1)^{\theta-1}, \quad z>0
$$

The general solution of the differential equation in Corollary 2.1.1 is

$$
\xi(z)=\exp \left[-1+(z \beta+1)^{\theta}\right]\left[-\int \beta \theta(z \beta+1)^{\theta-1} \boldsymbol{o}_{z ; \theta, \beta}\left[h_{1}(z)\right]^{-1} h_{2}(z)+D\right],
$$

where $D$ is a constant. Note that a set of functions satisfying the above differential equation is given in Proposition 2.1.1 with $D=0$. However, it should be also noted that there are other triplets $\left(h_{1}, h_{2}, \tau\right)$ satisfying the conditions of Theorem 2.1.1.

\subsection{Characterization based on HRF}

It is well known that the HRF, $h_{F}$, of a twice differentiable distribution function, $F$, satisfies the first order differential equation

$$
\frac{f^{\prime}(z)}{f(z)}=\frac{h_{F}^{\prime}(z)}{h_{F}(z)}-h_{F}(z)
$$

For many univariate continuous distributions, this is the only characterization available in terms of the HRF. The following proposition establishes a characterization of BXGNH distribution, for $a=1$, in terms of the HRF, which is not of the above trivial form.

\section{Proposition 2.2.1.}

Let $Z: \Omega \rightarrow(0,+\infty)$ be a continuous RV. The PDF of $Z$ is (4) if and only if its hazard function $h_{F}(z)$ satisfies the differential equation

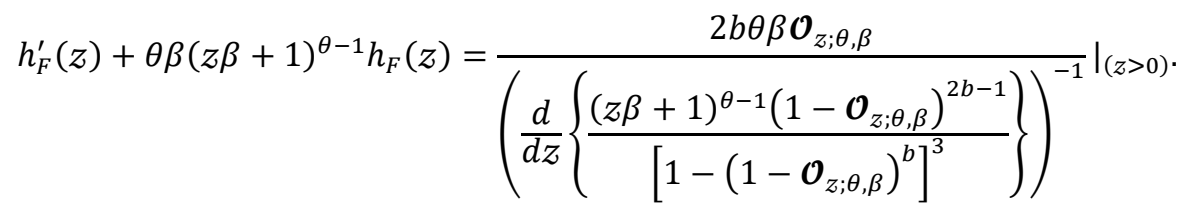

Proof. Is straightforward and hence omitted.

\subsection{Characterizations based on conditional expectation}

The following propositions have already appeared in Hamedani (2013), so we will just state them here which can be used to characterize the BXGNH distribution.

\section{Proposition 2.3.1.}

Let $Z: \Omega \rightarrow(a, b)$ be a continuous RV with CDF $F$. Let $\boldsymbol{\Phi}(z)$ be a differentiable function on $(a, b)$ with $\lim _{z \rightarrow a^{+}}[\boldsymbol{\Phi}(z)]=1$. Then for $\delta \neq 1$

if and only if

$$
\boldsymbol{E}[\boldsymbol{\Phi}(z) \mid Z \geq z]=\left.\delta \boldsymbol{\Phi}(Z)\right|_{(z \in(a, b))}
$$

$$
\boldsymbol{\Phi}(z)=\left.\left[1-F_{\underline{\Phi}}(z)\right]^{\delta^{-1}-1}\right|_{(z \in(a, b))}
$$

\section{Proposition 2.3.2.}

Let $Z: \Omega \rightarrow(a, b)$ be a continuous RV with $\operatorname{CDF} F$. Let $\boldsymbol{\Phi}_{1}(z)$ be a differentiable function on $(a, b)$ with $\lim _{z \rightarrow b^{-}}\left[\boldsymbol{\Phi}_{1}(z)\right]=1$. Then for $\delta_{1} \neq 1$, we have

implies that $\boldsymbol{\Phi}_{1}(z)=\left.\left[F_{\underline{\Phi}}(z)\right]^{\delta_{1}^{-1}-1}\right|_{(z \in(a, b))}$.

$$
\boldsymbol{E}\left[\boldsymbol{\Phi}_{1}(z) \mid Z \leq z\right]=\left.\delta \boldsymbol{\Phi}_{1}(z)\right|_{(z \in(a, b))}
$$

Remarks 2.3.1.

(A) For $(a, b)=(0,+\infty), a=1$, 


$$
\boldsymbol{\Phi}(z)=\exp \left[\left(\left\{1-\boldsymbol{O}_{z ; \theta, \beta}\right\}^{-b}-1\right)^{-2}\right]
$$

and $\delta=\frac{1}{2}$, Proposition 2.3.1 provides a characterization of BXGNH distribution.

(B) For $(a, b)=(0,+\infty)$,

$$
\boldsymbol{\Phi}_{1}(z)=1-\exp \left[\left(\left\{1-\boldsymbol{O}_{z ; \theta, \beta}\right\}^{-b}-1\right)^{-2}\right]
$$

and $\delta_{1}=\frac{a}{a+1}$, Proposition 2.3.2 provides a characterization of BXGNH distribution.

\section{Mathematical and statistical properties 3.1 Useful representation}

In this section, we provide a very useful linear representation for the BXGNH density function. If $\left|\frac{\omega_{1}}{\omega_{2}}\right|<1$ and $\omega_{3}>$ 0 is a real non-integer, the following power series holds

$$
\left(1-\frac{\omega_{1}}{\omega_{2}}\right)^{\omega_{3}}=\sum_{\omega_{4}=0}^{+\infty} \frac{(-1)^{\omega_{4}} \Gamma\left(1+\omega_{3}\right)}{\omega_{4} ! \Gamma\left(1+\omega_{3}-\omega_{4}\right)}\left(\frac{\omega_{1}}{\omega_{2}}\right)^{\omega_{4}}
$$

Applying (5) to term $\left(\left\{1-\exp \left[-\left(\left\{1-\boldsymbol{O}_{z ; \theta, \beta}\right\}^{-b}-1\right)^{-2}\right]\right\}\right)_{+\infty}^{a-1}$ in (4) we have

$$
\begin{aligned}
& f_{\underline{\Phi}}(z)=2 a b \theta \beta(z \beta+1)^{\theta-1} \boldsymbol{\mathcal { O }}_{z ; \theta, \beta} \sum_{\tau_{3}=0}^{+\infty} \frac{(-1)^{\tau_{3}} \Gamma(a)}{\tau_{3} ! \Gamma\left(a-\tau_{3}\right)} \\
& \times \frac{\left(1-\boldsymbol{\mathcal { O }}_{z ; \theta, \beta}\right)^{2 b-1}}{\left[1-\left(1-\boldsymbol{\mathcal { O }}_{z ; \theta, \beta}\right)^{b}\right]^{3}} \exp \left[-\left(1+\tau_{3}\right)\left(\left\{1-\boldsymbol{\mathcal { O }}_{z ; \theta, \beta}\right\}^{-b}-1\right)^{-2}\right] .
\end{aligned}
$$

Applying the power series to the term

equation (6) becomes

$$
\exp \left[-\left(1+\tau_{3}\right)\left(\left\{1-\boldsymbol{o}_{z ; \theta, \beta}\right\}^{-b}-1\right)^{-2}\right]
$$

$$
f_{\underline{\Phi}}(z)=2 a b \theta \beta \frac{\boldsymbol{o}_{z ; \theta, \beta}}{(z \beta+1)^{1-\theta}} \sum_{\tau_{1}, \tau_{3}=0}^{+\infty} \frac{(-1)^{\tau_{3}+\tau_{1}}\left(1+\tau_{3}\right)^{\tau_{1}} \Gamma(a)}{\tau_{3} ! \tau_{1} ! \Gamma\left(a-\tau_{3}\right)} \frac{\left(1-\boldsymbol{\mathcal { O }}_{z ; \theta, \beta}\right)^{b\left(2 \tau_{1}+2\right)-1}}{\left[1-\left(1-\boldsymbol{\mathcal { O }}_{z ; \theta, \beta}\right)^{b}\right]^{2 \tau_{1}+3}} .
$$

Consider the series expansion

$$
\left(1-\frac{\omega_{1}}{\omega_{2}}\right)^{-\omega_{3}}=\sum_{\omega_{4}=0}^{+\infty} \frac{\Gamma\left(\omega_{3}+\omega_{4}\right)}{\omega_{4} ! \Gamma\left(\omega_{3}\right)}\left(\frac{\omega_{1}}{\omega_{2}}\right)^{\omega_{4}} \mathrm{I}_{\left(\left|\frac{\omega_{1}}{\omega_{2}}\right|<1 \text { and } \omega_{3}>0\right)}
$$

Applying the expansion in (8) to (7) for the term $\left(1-\left\{1-\boldsymbol{O}_{z ; \theta, \beta}\right\}^{b}\right)^{2 \tau_{1}+3}$, equation (7) becomes

$$
f_{\underline{\Phi}}(z)=2 a \sum_{\tau_{1}, \tau_{2}, \tau_{3}=0}^{+\infty} \frac{(-1)^{\tau_{3}+\tau_{1}}\left(1+\tau_{3}\right)^{\tau_{1}} \Gamma(a) \Gamma\left(2 \tau_{1}+\tau_{2}+3\right)}{\tau_{3} ! \tau_{1} ! \tau_{2} ! \Gamma\left(a-\tau_{3}\right) \Gamma\left(2 \tau_{1}+3\right) \Delta} \underbrace{\left(\Delta \theta \beta(z \beta+1)^{\theta-1} \boldsymbol{O}_{z ; \theta, \beta}\left\{1-\boldsymbol{O}_{z ; \theta, \beta}\right\}^{\Delta-1}\right)}_{\pi_{\Delta, \theta, \beta}(z)},
$$

where $\boldsymbol{\Delta}=b\left(2+2 \tau_{1}+\tau_{2}\right)$. This can be written as

$$
f_{\underline{\Phi}}(z)=\sum_{\tau_{1}, \tau_{2}=0}^{+\infty} a_{\left(\tau_{1}, \tau_{2}\right)} \pi_{\Delta, \theta, \beta}(z)
$$

where

$$
a_{\left(\tau_{1}, \tau_{2}\right)}=\frac{2 a(-1)^{\tau_{1}} \Gamma(a) \Gamma\left(2 \tau_{1}+\tau_{2}+3\right)}{\tau_{1} ! \tau_{2} ! \Gamma\left(2 \tau_{1}+3\right)\left(2 \tau_{1}+\tau_{2}+2\right)} \sum_{\tau_{3}=0}^{+\infty} \frac{(-1)^{\tau_{3}}\left(\tau_{3}+1\right)^{\tau_{1}}}{\tau_{3} ! \Gamma\left(a-\tau_{3}\right)},
$$

and $\pi_{\Delta, \theta, \beta}(z)$ is the PDF of the GNH model with power parameter $\Delta$. equation (9) reveals that the density of $Z$ can be 
expressed as a linear mixture of GNH densities. So, several mathematical properties of the new family can be obtained from those of the GNH distribution. Similarly, the CDF of the BXGNH model can also be expressed as a mixture of GNH CDFs given by

$$
F_{\underline{\Phi}}(z)=\sum_{\tau_{1}, \tau_{2}=0}^{+\infty} a_{\left(\tau_{1}, \tau_{2}\right)} \boldsymbol{\Pi}_{\Delta, \theta, \beta}(z),
$$

where $\boldsymbol{\Pi}_{\boldsymbol{\Delta}, \theta, \beta}(z)=\left(1-\boldsymbol{\mathcal { O }}_{z ; \theta, \beta}\right)^{\boldsymbol{\Delta}}$ is the CDF of the GNH model with power parameter $\boldsymbol{\Delta}$.

\subsection{Moments and moment generating function}

The $r^{\text {th }}$ ordinary moment of $Z$ is given by $\mu_{r, Z}^{\prime}=\boldsymbol{E}\left(Z^{r}\right)=\int_{-\infty}^{+\infty} f_{\underline{\Phi}}(z) z^{r} d z$. Then we obtain

$$
\mu_{r, Z}^{\prime}=\sum_{\tau_{1}, \tau_{2}, \tau_{3}=0}^{+\infty} \sum_{\tau_{4}=0}^{r} C_{\left(\tau_{1}, \tau_{2}, \tau_{3}, \tau_{4}\right)}^{[\Delta, r]} \Gamma\left(1+\frac{\tau_{4}}{\theta}, 1+\tau_{3}\right)
$$

where

Or

$$
\begin{gathered}
C_{\left(\tau_{1}, \tau_{2}, \tau_{3}, \tau_{4}\right)}^{[\Delta,]}=a_{\left(\tau_{1}, \tau_{2}\right)} C_{\tau_{3}, \tau_{4},}^{[\Delta, r]} \\
C_{\tau_{3}, \tau_{4}}^{[\eta, r]}=\eta \beta^{-r} \frac{(-1)^{r+\tau_{3}-\tau_{4}} \exp \left(1+\tau_{3}\right)}{\left(1+\tau_{3}\right)^{1+\eta^{-1} \tau_{4}}}\left(\begin{array}{c}
r \\
\tau_{4}
\end{array}\right)\left(\begin{array}{c}
-1+\eta \\
\tau_{3}
\end{array}\right) .
\end{gathered}
$$

$$
\mu_{r, Z}^{\prime}=\left.\sum_{\tau_{1}, \tau_{2}=0}^{+\infty} \sum_{\tau_{3}=0}^{\Delta-1} \sum_{\tau_{4}=0}^{r} C_{\left(\tau_{1}, \tau_{2}, \tau_{3}, \tau_{4}\right)}^{[\Delta, r]} \Gamma\left(1+\frac{\tau_{4}}{\theta}, 1+\tau_{3}\right)\right|_{(\Delta>0 \text { and integer })} .
$$

Setting $r=1$ in (11), we have the mean of $z$

where $C_{\left(\tau_{1}, \tau_{2}, \tau_{3}, \tau_{4}\right)}^{[\Delta, 1]}=a_{\left(\tau_{1}, \tau_{2}\right)} C_{\tau_{3}, \tau_{4}}^{[\Delta, 1]}$ and

$$
\mu_{1, Z}^{\prime}=\sum_{\tau_{1}, \tau_{2}, \tau_{3}=0}^{+\infty} \sum_{\tau_{4}=0}^{1} C_{\left(\tau_{1}, \tau_{2}, \tau_{3}, \tau_{4}\right)}^{[\Delta, 1]} \Gamma\left(1+\frac{\tau_{4}}{\theta}, 1+\tau_{3}\right),
$$

Then,

$$
C_{\tau_{3}, \tau_{4}}^{[a, 1]}=\frac{a}{\beta} \frac{(-1)^{r+\tau_{3}-\tau_{4}} \exp \left(1+\tau_{3}\right)}{\left(1+\tau_{3}\right)^{1+\frac{\tau_{4}}{\theta}}}\left(\begin{array}{c}
-1+a \\
\tau_{3}
\end{array}\right)\left(\begin{array}{c}
1 \\
\tau_{4}
\end{array}\right) .
$$

$$
\mu_{r, Z}^{\prime}=\left.\sum_{\tau_{1}, \tau_{2}=0}^{+\infty} \sum_{\tau_{3}=0}^{\Delta-1} \sum_{\tau_{4}=0}^{1} C_{\left(\tau_{1}, \tau_{2}, \tau_{3}, \tau_{4}\right)}^{(\Delta, 1)} \Gamma\left(1+\frac{\tau_{4}}{\theta}, 1+\tau_{3}\right)\right|_{(\Delta>0 \text { and integer })},
$$

where $\Gamma(a, v)=\int_{v}^{+\infty} z^{-1+a} \exp (-z) d z$ denotes the complementary incomplete gamma function, which can be evaluated in MATHEMATICA, R, etc. The variance $(\operatorname{Var}(Z))$, skewness $(\operatorname{Ske}(Z))$ and kurtosis $(\operatorname{Ku}(Z))$ measures can be calculated from the ordinary moments using well-known relationships (see the numerical analysis given in Tables $1 \& 2$ ). Here, we provide a formula for the moment generating function (MGF) $M_{Z}(t)=\boldsymbol{E}\left(e^{t Z}\right)$ of $Z$. Clearly, the MGF can be derived from (9) as

and

$$
M_{Z}(t)=\sum_{\tau_{1}, \tau_{2}, \tau_{3}, r=0}^{+\infty} \sum_{\tau_{4}=0}^{r} \frac{t^{r}}{r !} C_{\left(\tau_{1}, \tau_{2}, \tau_{3}, \tau_{4}\right)}^{\left[\Delta, \tau_{1}\right.} \Gamma\left(1+\frac{\tau_{4}}{\theta}, 1+\tau_{3}\right),
$$

$$
M_{Z}(t)=\left.\sum_{\tau_{1}, \tau_{2}, r=0}^{+\infty} \sum_{\tau_{3}=0}^{\Delta-1} \sum_{\tau_{4}=0}^{r} \frac{t^{r}}{r !} C_{\left(\tau_{1}, \tau_{2}, \tau_{3}, \tau_{4}\right)}^{[\Delta, r]} \Gamma\left(1+\frac{\tau_{4}}{\theta}, 1+\tau_{3}\right)\right|_{(\Delta>0 \text { and integer })} .
$$

\subsection{Incomplete moments}

The $s^{\text {th }}$ incomplete moment, say $\boldsymbol{Q}_{s, Z}(t)$, of $Z$ can be expressed from (9) as 


$$
\boldsymbol{Q}_{s, Z}(t)=\sum_{\tau_{1}, \tau_{2}, \tau_{3}=0}^{+\infty} \sum_{\tau_{4}=0}^{s} C_{\left(\tau_{1}, \tau_{2}, \tau_{3}, \tau_{4}\right)}^{[\Lambda, s]}\left[\begin{array}{c}
\Gamma\left(1+\frac{\tau_{4}}{\theta}, 1+\tau_{3}\right) \\
-\Gamma\left(1+\frac{\tau_{4}}{\theta},\left(1+\tau_{3}\right)(1+\beta t)^{\theta}\right)
\end{array}\right],
$$

and

$$
\boldsymbol{Q}_{s, Z}(t)=\sum_{\tau_{1}, \tau_{2}=0}^{+\infty} \sum_{\tau_{3}=0}^{\Delta-1} \sum_{\tau_{4}=0}^{s} C_{\left(\tau_{1}, \tau_{2}, \tau_{3}, \tau_{4}\right)}^{[\Delta, s]}\left[\begin{array}{c}
\Gamma\left(1+\frac{\tau_{4}}{\theta}, 1+\tau_{3}\right) \\
-\Gamma\left(1+\frac{\tau_{4}}{\theta},\left(1+\tau_{3}\right)(1+\beta t)^{\theta}\right)
\end{array}\right] I_{(\Delta>0 \text { and integer })} .
$$

The first incomplete moment, $\boldsymbol{Q}_{1, Z}(t)$, can be derived from (12) as

$$
\mathcal{Q}_{1, Z}(t)=\sum_{\tau_{1}, \tau_{2}, \tau_{3}=0}^{+\infty} \sum_{\tau_{4}=0}^{1} C_{\left(\tau_{1}, \tau_{2}, \tau_{3}, \tau_{4}\right)}^{[\Delta, 1]}\left[\begin{array}{c}
\Gamma\left(1+\frac{\tau_{4}}{\theta}, 1+\tau_{3}\right) \\
-\Gamma\left(1+\frac{\tau_{4}}{\theta},\left(1+\tau_{3}\right)(1+\beta t)^{\theta}\right)
\end{array}\right],
$$

and

$$
\boldsymbol{Q}_{1, Z}(t)=\sum_{\tau_{1}, \tau_{2}=0}^{+\infty} \sum_{\tau_{3}=0}^{\Delta-1} \sum_{\tau_{4}=0}^{1} C_{\left(\tau_{1}, \tau_{2}, \tau_{3}, \tau_{4}\right)}^{[\Delta, 1]}\left[\begin{array}{c}
\Gamma\left(1+\frac{\tau_{4}}{\theta}, 1+\tau_{3}\right) \\
-\Gamma\left(1+\frac{\tau_{4}}{\theta},\left(1+\tau_{3}\right)(1+\beta t)^{\theta}\right)
\end{array}\right] I_{(\Delta>0 \text { and integer })} .
$$

\subsection{Probability weighted moments (PWMs)}

The $(s, r)^{\text {th }}$ PWM of $Z$ with the BXGNH model, say $\mu_{s, r}(Z)$, is formally defined by

$$
\mu_{s, r}(Z)=\boldsymbol{E}\left\{Z^{s} F_{\underline{\Phi}}(Z)^{r}\right\}=\int_{-\infty}^{+\infty} Z^{s} F_{\underline{\Phi}}(z)^{r} f_{\underline{\Phi}}(z) d z .
$$

Using equations (3) and (4) we can write $f_{\underline{\Phi}}(z) F_{\underline{\Phi}}(z)^{r}=\sum_{\tau_{1}, \tau_{2}=0}^{+\infty} q_{\left(\tau_{1}, \tau_{2}\right)} \pi_{[\Delta], \theta, \beta}(z)$ where

$$
q_{\left(\tau_{1}, \tau_{2}\right)}=\frac{2 a(-1)^{\tau_{1}} \Gamma\left(2 \tau_{1}+\tau_{2}+3\right)}{\tau_{1} ! \tau_{2} ! \Gamma\left(2 \tau_{1}+3\right) \Delta} \sum_{m=0}^{+\infty}(-1)^{m}(1+m)^{\tau_{1}}\left(\begin{array}{c}
a(r+1)-1 \\
m
\end{array}\right) .
$$

Then, the $(s, r)^{\text {th }}$ PWM of $Z$ can be expressed as

where $V_{\tau_{3}, \tau_{4}}^{[\Lambda, s]}=q_{\left(\tau_{1}, \tau_{2}\right)} C_{\tau_{3}, \tau_{4}}^{[\Delta, s]}$ and also

$$
\mu_{s, r}(Z)=\sum_{\tau_{1}, \tau_{2}, \tau_{3}=0}^{+\infty} \sum_{\tau_{4}=0}^{r} V_{\tau_{3}, \tau_{4}}^{[\Delta, s]} \Gamma\left(1+\frac{\tau_{4}}{\theta}, 1+\tau_{3}\right),
$$

$$
\mu_{s, r}(Z)=\left.\sum_{\tau_{1}, \tau_{2}=0}^{+\infty} \sum_{\tau_{3}=0}^{\Delta-1} \sum_{\tau_{4}=0}^{r} V_{\tau_{3}, \tau_{4}}^{[\Delta, s]} \Gamma\left(1+\frac{\tau_{4}}{\theta}, 1+\tau_{3}\right)\right|_{(\Delta>0 \text { and integer })} .
$$

\subsection{Residual and reversed residual life}

The $n^{\text {th }}$ moment of the residual life is

$$
a_{n, Z}(t)=\left.\boldsymbol{E}\left[(Z-t)^{n}\right]\right|_{Z>t \text { and } n=1,2, \ldots}
$$

The $n^{\text {th }}$ moment of the residual life of $Z$ is given by

Therefore

$$
a_{n, Z}(t)=\frac{\int_{t}^{+\infty}(z-t)^{n} d F_{\underline{\Phi}}(z)}{1-F(t)}
$$

or

$$
a_{n, Z}(t)=\frac{1}{1-F(t)} \sum_{\tau_{1}, \tau_{2}, \tau_{3}=0}^{+\infty} \sum_{\tau_{4}=0}^{n} \sum_{r=0}^{n}\left(\begin{array}{l}
n \\
r
\end{array}\right)(-t)^{n-r} C_{\left(\tau_{1}, \tau_{2}, \tau_{3}, \tau_{4}\right)}^{[\Delta, n]} \Gamma\left(1+\frac{\tau_{4}}{\theta}, 1+\tau_{3}\right)
$$

$$
a_{n, Z}(t)=\left.\frac{1}{1-F(t)} \sum_{\tau_{1}, \tau_{2}=0}^{+\infty} \sum_{\tau_{3}=0}^{\Delta-1} \sum_{\tau_{4}=0}^{n} \sum_{r=0}^{n}\left(\begin{array}{l}
n \\
r
\end{array}\right)(-t)^{n-r} C_{\left(\tau_{1}, \tau_{2}, \tau_{3}, \tau_{4}\right)}^{[[, n]} \Gamma\left(1+\frac{\tau_{4}}{\theta}, 1+\tau_{3}\right)\right|_{(\Delta>0 \text { and integer })} .
$$

The $n^{\text {th }}$ moment of the reversed residual life is 
then, we obtain

$$
A_{n, Z}(t)=\left.\boldsymbol{E}\left[(t-Z)^{n}\right]\right|_{(Z \leq t, t>0 \text { and } n=1,2, \ldots)},
$$

$$
A_{n, Z}(t)=\frac{1}{F(t)} \int_{0}^{t}(t-z)^{n} d F_{\underline{\Phi}}(z) .
$$

Then, the $n^{\text {th }}$ moment of the reversed residual life of $Z$ becomes

$$
A_{n, Z}(t)=\frac{1}{F(t)} \sum_{\tau_{1}, \tau_{2}, \tau_{3}=0}^{+\infty} \sum_{\tau_{4}=0}^{n} \sum_{r=0}^{n}(-1)^{r}\left(\begin{array}{l}
n \\
r
\end{array}\right) t^{n-r} C_{\left(\tau_{1}, \tau_{2}, \tau_{3}, \tau_{4}\right)}^{[\Delta, n]}\left[\begin{array}{c}
\Gamma\left(1+\frac{\tau_{4}}{\theta}, 1+\tau_{3}\right) \\
-\Gamma\left(1+\frac{\tau_{4}}{\theta},\left(1+\tau_{3}\right)(1+\beta t)^{\theta}\right)
\end{array}\right]
$$

or

$$
A_{n, Z}(t)=\frac{1}{F(t)} \sum_{\tau_{1}, \tau_{2}=0}^{+\infty} \sum_{\tau_{3}=0}^{\Delta-1} \sum_{\tau_{4}=0}^{n} \sum_{r=0}^{n}(-1)^{r}\left(\begin{array}{c}
n \\
r
\end{array}\right) t^{n-r} C_{\left(\tau_{1}, \tau_{2}, \tau_{3}, \tau_{4}\right)}^{[\Delta, n]}\left[\begin{array}{c}
\Gamma\left(1+\frac{\tau_{4}}{\theta}, 1+\tau_{3}\right) \\
-\Gamma\left(1+\frac{\tau_{4}}{\theta},\left(1+\tau_{3}\right)(1+\beta t)^{\theta}\right)
\end{array}\right] I_{(\Delta>0 \text { and integer })}
$$

\subsection{Order statistics}

Let $Z_{(1)}, Z_{(2)}, \ldots, Z_{(n)}$ be a random sample (RS) from the BXGNH distribution and $Z_{1, n}, Z_{2, n}, \ldots, Z_{n, n}$ be the corresponding order statistics. The PDF of the $i^{\text {th }}$ order statistic, say $Z_{i: n}$, can be written as

$$
f_{(i: n)}(z)=\frac{f_{\underline{\Phi}}(z)}{B(i, n-i+1)} \sum_{j=0}^{n-i}(-1)^{j}\left(\begin{array}{c}
n-i \\
j
\end{array}\right) F^{j+i-1}(z),
$$

where $B(\cdot, \cdot)$ refers to the beta function. Using (3), (4) in equation (13) we get

$$
f_{\underline{\Phi}}(z) F_{\underline{\Phi}}(z)^{j+i-1}=\sum_{\tau_{1}, \tau_{2}=0}^{+\infty} r_{\left(\tau_{1}, \tau_{2}\right)} \pi_{\Delta, \theta, \beta}(z)
$$

where

$$
r_{\left(\tau_{1}, \tau_{2}\right)}=\frac{2 a(-1)^{\tau_{1}} \Gamma\left(2 \tau_{1}+\tau_{2}+3\right)}{\tau_{1} ! \tau_{2} ! \Gamma\left(2 \tau_{1}+3\right) \Delta} \sum_{m=0}^{+\infty}(-1)^{m}(1+m)^{\tau_{1}}\left(\begin{array}{c}
a(j+i)-1 \\
m
\end{array}\right)
$$

The PDF of $Z_{(i: n)}$ can be expressed as

$$
f_{(i: n)}(z)=\sum_{\tau_{1}, \tau_{2}=0}^{+\infty} \sum_{j=0}^{n-i}(-1)^{j} \frac{r_{\left(\tau_{1}, \tau_{2}\right)}}{B(i, n-i+1)}\left(\begin{array}{c}
n-i \\
j
\end{array}\right) \pi_{\Delta, \theta, \beta}(z) .
$$

Then, the density function of the BXGNH order statistic is a mixture of GNH density. Based on (14), the moments of $Z_{(i: n)}$ can be expressed as

where

$$
\boldsymbol{E}\left(Z_{(i: n)}^{q}\right)=\sum_{\tau_{1}, \tau_{2}, \tau_{3}=0}^{+\infty} \sum_{\tau_{4}=0}^{r} \sum_{j=0}^{n-i}(-1)^{j} \frac{W_{\left(\tau_{1}, \tau_{2}, \tau_{3}, \tau_{4}\right)}^{[\Delta, q]}}{B(i, n-i+1)}\left(\begin{array}{c}
n-i \\
j
\end{array}\right) \Gamma\left(1+\frac{\tau_{4}}{\theta}, 1+\tau_{3}\right),
$$

Or

$$
W_{\left(\tau_{1}, \tau_{2}, \tau_{3}, \tau_{4}\right)}^{[\Delta, q]}=r_{\left(\tau_{1}, \tau_{2}\right)} C_{\tau_{3}, \tau_{4}}^{[\Delta, q]}
$$

$$
\boldsymbol{E}\left(Z_{(i: n)}^{q}\right)=\left.\sum_{\tau_{1}, \tau_{2}=0}^{+\infty} \sum_{\tau_{3}=0}^{\Delta-1} \sum_{\tau_{4}=0}^{r} \sum_{j=0}^{n-i}(-1)^{j} \frac{W_{\left(\tau_{1}, \tau_{2}, \tau_{3}, \tau_{4}\right)}^{[\Delta, q]}}{B(i, n-i+1)}\left(\begin{array}{c}
n-i \\
j
\end{array}\right) \Gamma\left(1+\frac{\tau_{4}}{\theta}, 1+\tau_{3}\right)\right|_{(\Delta>0 \text { and integer })} .
$$

\subsection{Numerical analysis for certain measures}

Numerical analysis for the $\boldsymbol{E}(Z), \operatorname{Var}(Z), \operatorname{Ske}(Z)$ and $\mathrm{Ku}(Z)$ is presented in Table 1 for the BXGNH and in Table 2 for the GNH model (see Tables 1 and 2 in the Appendix). Based on Table 1 we note that the skewness of the BXGNH distribution is always positive. The kurtosis of the BXGNH distribution can be only more than 3 . The parameter $\beta$ has a fixed effect on the $\operatorname{Ske}(Z)$ and $\operatorname{Ku}(Z)$ for all different values of all other parameter. When $a=0.5, b=0.1$ and $\theta$ $=0.05, \operatorname{Ske}(Z)=6.95$ and $\operatorname{Ku}(Z)=82.94$ for any value of the parameter $\beta$. when $a=1.5, b=1.25$ and $\theta=0.2, \operatorname{Ske}(Z)$ $=1.213746$ and $\mathrm{Ku}(Z)=5.033408$ for any value of the parameter $\beta$. The mean of the BXGNH distribution increases 
as $a$ increases. The mean of the BXGNH distribution increases as $b$ increases. The mean of the proposed model decreases as $\theta$ increases. The mean of the proposed model decreases as $\beta$ increases. Based on Tables 1 and 2 we note that, the skewness of the BXGNH distribution can range in the interval $(0.86,6.96)$, whereas the skewness of the GNH distribution varies only in the interval $(0.435,3.17)$. Further, the spread for the BXGNH kurtosis is ranging from 4.61 to 1225.3 , whereas the spread for the GNH kurtosis only varies only from 3.37 to 9 with the above parameter values.

Table 1: $\boldsymbol{E}(\mathrm{Z}), \operatorname{Var}(\mathrm{Z})$, Ske(Z) and $\mathrm{Ku}(\mathrm{Z})$ of the BXGNH distribution.

\begin{tabular}{|c|c|c|c|c|c|c|c|}
\hline $\mathrm{a}$ & $\mathrm{b}$ & $\theta$ & $\beta$ & $\boldsymbol{E}(\mathrm{Z})$ & $\operatorname{Var}(\mathrm{Z})$ & $\operatorname{Ske}(Z)$ & $\mathrm{Ku}(\mathrm{Z})$ \\
\hline 0.5 & 3 & 0.15 & 100 & 3.363791 & 14.47751 & 2.0623060 & 8.751728 \\
\hline 1 & & & & 5.228350 & 18.76705 & 1.5520220 & 6.331646 \\
\hline 5 & & & & 10.81115 & 22.5588 & 1.0408350 & 4.738005 \\
\hline 10 & & & & 13.39890 & 22.16723 & 0.9803191 & 4.625609 \\
\hline 25 & & & & 16.79086 & 21.05432 & 0.9562387 & 4.606228 \\
\hline 50 & & & & 19.30014 & 20.10823 & 0.9566659 & 4.628752 \\
\hline 100 & & & & 21.75179 & 19.19630 & 0.9632528 & 4.661848 \\
\hline 250 & & & & 24.90502 & 18.10005 & 0.9749314 & 4.708858 \\
\hline 500 & & & & 27.22813 & 17.35974 & 0.9839197 & 4.742866 \\
\hline 1000 & & & & 29.50197 & 16.69005 & 0.992346 & 4.774471 \\
\hline \multirow[t]{6}{*}{10} & 0.25 & 0.1 & 10 & 0.425889 & 0.088030 & 2.593693 & 16.46716 \\
\hline & 0.5 & & & 6.648164 & 25.94129 & 2.701086 & 17.04928 \\
\hline & 1 & & & 109.3432 & 6024.079 & 2.280805 & 12.57559 \\
\hline & 1.5 & & & 501.6328 & 105562.5 & 2.005222 & 10.25414 \\
\hline & 2 & & & 1375.197 & 686032.2 & 1.828003 & 8.960356 \\
\hline & 2.25 & & & 2041.229 & 1422021 & 1.760982 & 8.50895 \\
\hline \multirow[t]{6}{*}{2.5} & 0.25 & 0.05 & 5 & 2.6017280 & 44.605390 & 18.50410 & 1225.323 \\
\hline & & 0.1 & & 0.4098065 & 0.1884818 & 3.320510 & 24.67575 \\
\hline & & 0.15 & & 0.2040524 & 0.0294352 & 2.064140 & 10.34501 \\
\hline & & 0.2 & & 0.1341438 & 0.0103092 & 1.625269 & 7.222300 \\
\hline & & 0.3 & & 0.0790552 & 0.0029387 & 1.265282 & 5.290963 \\
\hline & & 0.4 & & 0.0559123 & 0.0013377 & 1.107794 & 4.611152 \\
\hline \multirow[t]{4}{*}{0.5} & 0.1 & 0.05 & 0.1 & 0.2439578 & 0.5573194 & 6.955959 & 82.93746 \\
\hline & & & 0.5 & 0.0243958 & 0.0055732 & 6.955994 & 82.93802 \\
\hline & & & 1 & 0.0121982 & 0.0013933 & 6.956147 & 82.94034 \\
\hline & & & 2 & 0.0060989 & 0.0003483 & 6.956633 & 82.94702 \\
\hline \multirow[t]{4}{*}{1.5} & 1.25 & 0.2 & 1 & 24.02387 & 238.0755 & 1.213746 & 5.033408 \\
\hline & & & 2 & 12.01193 & 59.51887 & 1.213746 & 5.033408 \\
\hline & & & 3 & 8.007955 & 26.45283 & 1.213746 & 5.033408 \\
\hline & & & 4 & 6.005966 & 14.87972 & 1.213746 & 5.033408 \\
\hline 3 & 2 & 0.2 & 2 & 39.472250 & 299.1818 & 0.860440 & 4.056376 \\
\hline 10 & 0.5 & 0.1 & 10 & 6.6481640 & 25.94129 & 2.701086 & 17.04928 \\
\hline 1 & 1 & 0.25 & 1 & 6.5601690 & 19.78288 & 1.110063 & 4.475175 \\
\hline 0.1 & 0.1 & 0.25 & 0.1 & 0.0049732 & 0.001025 & 13.10943 & 259.3826 \\
\hline 4 & 1.25 & 0.1 & 1.5 & 1072.3240 & 944208.8 & 2.558604 & 14.59917 \\
\hline 0.5 & 0.25 & 0.1 & 0.5 & 1.2602790 & 6.575796 & 5.478182 & 59.79648 \\
\hline
\end{tabular}


Table 2: $\boldsymbol{E}(\mathrm{Z}), \operatorname{Var}(\mathrm{Z}), \mathrm{Ske}(\mathrm{Z})$ and $\mathrm{Ku}(\mathrm{Z})$ of the $\mathrm{GNH}$ distribution.

\begin{tabular}{|c|c|c|c|c|c|c|}
\hline$\theta$ & $\mathrm{a}$ & $\mathrm{b}$ & $\boldsymbol{E}(\mathrm{Z})$ & $\operatorname{Var}(\mathrm{Z})$ & Ske(Z) & $\mathrm{Ku}(\mathrm{Z})$ \\
\hline 1 & 1 & 1 & 1 & 1 & 2 & 9 \\
\hline 2 & & & 1.5 & 1.25 & 1.609969 & 7.08 \\
\hline 5 & & & 2.283333 & 1.463611 & 1.339221 & 6.025973 \\
\hline 10 & & & 2.928968 & 1.549768 & 1.241416 & 5.703086 \\
\hline 20 & & & 3.597740 & 1.596163 & 1.190993 & 5.548813 \\
\hline 50 & & & 4.499205 & 1.625133 & 1.160248 & 5.458834 \\
\hline 75 & & & 4.901356 & 1.631689 & 1.153366 & 5.439116 \\
\hline 100 & & & 5.187378 & 1.634984 & 1.149918 & 5.429296 \\
\hline 200 & & & 5.878031 & 1.639947 & 1.144738 & 5.414611 \\
\hline 500 & & & 6.792823 & 1.642936 & 1.141626 & 5.405815 \\
\hline \multirow[t]{5}{*}{5} & 0.5 & 5 & 2.2487780 & 4.20089400 & 3.176333 & 22.94556 \\
\hline & 1 & & 0.4566667 & 0.05854444 & 1.339221 & 6.025973 \\
\hline & 2 & & 0.1568573 & 0.00398623 & 0.743554 & 3.776470 \\
\hline & 3 & & 0.0932310 & 0.00117149 & 0.568839 & 3.366604 \\
\hline & 5 & & 0.0512124 & 0.00030503 & 0.435122 & 6.044819 \\
\hline \multirow[t]{4}{*}{1.5} & 2 & 0.1 & 4.750170 & 10.469720 & 1.04087 & 4.228012 \\
\hline & & 0.5 & 0.950034 & 0.4187887 & 1.04087 & 4.228012 \\
\hline & & 1 & 0.475017 & 0.1046971 & 1.04087 & 4.228012 \\
\hline & & 5 & 0.095003 & 0.0041879 & 1.04087 & 4.228012 \\
\hline 2.5 & 2.5 & 4 & 0.1137808 & 0.00342470 & 0.7489036 & 3.576191 \\
\hline 2 & 2 & 3 & 0.1823958 & 0.01180120 & 0.9293953 & 4.007148 \\
\hline 1 & 2 & 4 & 0.0947340 & 0.00615846 & 1.253913 & 4.772774 \\
\hline 4 & 2 & 1 & 0.7265553 & 0.10234000 & 0.7698508 & 3.792370 \\
\hline 0.5 & 2.5 & 1 & 0.1828302 & 0.04319620 & 1.664298 & 6.044604 \\
\hline 1.5 & 2.5 & 5 & 0.0719500 & 0.00222103 & 0.923067 & 3.827275 \\
\hline
\end{tabular}

\section{Non-Bayesian estimation methods \\ 4.1 MLE method}

Let $Z_{1}, Z_{2}, \ldots, Z_{n}$ be an observed RS from BXGNH distribution with parameter vector $\underline{\Phi}=(a, b, \theta, \beta)^{T}$. The $\log$ likelihood function for $\underline{\boldsymbol{\Phi}}$, say $\ell(\underline{\boldsymbol{\Phi}})$, is given by

$$
\begin{aligned}
& \ell \underline{\boldsymbol{\Phi}})=n \log a+n \log b+n \log \theta+n \log \beta-(b+1) \sum_{i=0}^{n} \log \left\{1-\boldsymbol{\mathcal { O }}_{\left[z_{i} ; \theta, \beta\right]}\right\} \\
& +n \log 2+(a-1) \sum_{i=0}^{n} \log \left(1-\exp \left\{-\left[\left(1-\boldsymbol{\mathcal { O }}_{\left[z_{i} ; \theta, \beta\right]}\right)^{-b}-1\right]^{-2}\right\}\right) \\
& +\sum_{i=0}^{n}\left[(z \beta+1)^{\theta}-1\right]-\sum_{i=0}^{n}\left[\left(1-\boldsymbol{\mathcal { O }}_{\left[z_{i} ; \theta, \beta\right]}\right)^{-b}-1\right]^{-2} .
\end{aligned}
$$

where $\ell(\underline{\Phi})$ can be maximized either by using the different programs like R (optim function), SAS (PROC NLMIXED) or by solving the nonlinear likelihood equations obtained by differentiating (15). The score vector elements $U(\underline{\boldsymbol{\Phi}})=\left(\frac{\partial \ell(\underline{\boldsymbol{\Phi}})}{\partial a}, \frac{\partial \ell(\underline{\boldsymbol{\Phi}})}{\partial b}, \frac{\partial \ell(\underline{\boldsymbol{\Phi}})}{\partial \theta}, \frac{\partial \ell(\underline{\boldsymbol{\Phi}})}{\partial \beta}\right)^{T}$ are easily to be derived.

\subsection{The CVME method}

The CVME of the parameters $a, b, \theta$ and $\beta$ are obtained via minimizing the following expression with respect to (WRT) to the parameters $a, b, \theta$ and $\beta$ respectively, where

$$
\left.F_{\underline{\Phi}}(z)\right|_{(z \geq 0)}=\left\{1-\exp \left[-\left(\left\{1-\mathcal{O}_{\left[z_{[i: n]} ; \theta, \beta\right]}\right\}^{-b}-1\right)^{-2}\right]\right\}^{a}
$$


where $v_{(i, n)}^{[1]}=\frac{2 i-1}{2 n}$ and

$$
C V M_{(\underline{\Phi})}=\frac{1}{12} n^{-1}+\sum_{i=1}^{n}\left[F_{\underline{\Phi}}\left(z_{[i: n]}\right)-v_{(i, n)}^{[1]}\right]^{2}
$$

$$
C V M_{(\underline{\Phi})}=\sum_{i=1}^{n}\left(\left\{1-\exp \left[-\left(\left\{1-\boldsymbol{o}_{\left[z_{[i: n]} ; \theta, \beta\right]}\right\}^{-b}-1\right)^{-2}\right]\right\}^{a}-v_{(i, n)}^{[1]}\right)^{2} .
$$

The, CVME of the parameters $a, b, \theta$ and $\beta$ are obtained by solving the two following non-linear equations

$$
\begin{aligned}
& \sum_{i=1}^{n}\left(\left\{1-\exp \left[-\left(\left\{1-\boldsymbol{\mathcal { O }}_{\left[z_{[i: n]} ; \theta, \beta\right]}\right\}^{-b}-1\right)^{-2}\right]\right\}^{a}-v_{(i, n)}^{[1]}\right) \delta_{a}\left(z_{[i: n]}, \underline{\boldsymbol{\Phi}}\right)=0 \\
& \sum_{i=1}^{n}\left(\left\{1-\exp \left[-\left(\left\{1-\boldsymbol{\mathcal { O }}_{\left[z_{[i: n]} ; \theta, \beta\right]}\right\}^{-b}-1\right)^{-2}\right]\right\}^{a}-v_{(i, n)}^{[1]}\right) \delta_{b}\left(z_{[i: n]}, \underline{\boldsymbol{\Phi}}\right)=0 \\
& \sum_{i=1}^{n}\left(\left\{1-\exp \left[-\left(\left\{1-\boldsymbol{\mathcal { O }}_{\left[z_{[i: n]} ; \theta, \beta\right]}\right\}^{-b}-1\right)^{-2}\right]\right\}^{a}-v_{(i, n)}^{[1]}\right) \delta_{\theta}\left(z_{[i: n]}, \underline{\boldsymbol{\Phi}}\right)=0
\end{aligned}
$$

and

$$
\sum_{i=1}^{n}\left(\left\{1-\exp \left[-\left(\left\{1-\boldsymbol{\mathcal { O }}_{\left[z_{[i: n]} ; \theta, \beta\right]}\right\}^{-b}-1\right)^{-2}\right]\right\}^{a}-v_{(i, n)}^{[1]}\right) \delta_{\beta}\left(z_{[i: n]}, \underline{\boldsymbol{\Phi}}\right)=0,
$$

where $\delta_{a}\left(z_{[i: n]}, \underline{\boldsymbol{\Phi}}\right), \delta_{b}\left(z_{[i: n]}, \underline{\boldsymbol{\Phi}}\right), \delta_{b}\left(z_{[i: n]}, \underline{\boldsymbol{\Phi}}\right)$ and $\delta_{\beta}\left(z_{[i: n]}, \underline{\boldsymbol{\Phi}}\right)$ are the first derivatives of the CDF of BXGNH distribution WRT $a, b, \theta$ and $\beta$ respectively.

\subsection{The OLS method}

Let $F_{\underline{\Phi}}\left(z_{[i: n]}\right)$ denotes the CDF of BXGNH model and let $Z_{1}<Z_{2}<\cdots<Z_{n}$ be the $n$ ordered RS. The OLSEs are obtained upon minimizing

then, we have

$$
\operatorname{OLSE}(\underline{\boldsymbol{\Phi}})=\sum_{i=1}^{n}\left[F_{\underline{\Phi}}\left(z_{[i: n]}\right)-v_{(i, n)}^{[2]}\right]^{2}
$$

$$
\operatorname{OLSE}(\underline{\boldsymbol{\Phi}})=\sum_{i=1}^{n}\left[\left\{1-\exp \left[-\left(\left\{1-\boldsymbol{\mathcal { O }}_{\left[z_{[i: n]} ; \theta, \beta\right]}\right\}^{-b}-1\right)^{-2}\right]\right\}^{a}-v_{(i, n)}^{[2]}\right]^{2},
$$

where $v_{(i, n)}^{[2]}=\frac{i}{n+1}$. The LSEs are obtained via solving the following non-linear equations

and

$$
\begin{aligned}
& 0=\sum_{i=1}^{n}\left[\left\{1-\exp \left[-\left(\left\{1-\boldsymbol{\mathcal { O }}_{\left[z_{[i: n]} ; \theta, \beta\right]}\right\}^{-b}-1\right)^{-2}\right]\right\}^{a}-v_{(i, n)}^{[2]}\right] \delta_{a}\left(z_{[i: n]}, \underline{\boldsymbol{\Phi}}\right), \\
& 0=\sum_{i=1}^{n}\left[\left\{1-\exp \left[-\left(\left\{1-\boldsymbol{\mathcal { O }}_{\left[z_{[i: n]} ; \theta, \beta\right]}\right\}^{-b}-1\right)^{-2}\right]\right\}^{a}-v_{(i, n)}^{[2]}\right] \delta_{b}\left(z_{[i: n]}, \underline{\boldsymbol{\Phi}}\right), \\
& 0=\sum_{i=1}^{n}\left[\left\{1-\exp \left[-\left(\left\{1-\boldsymbol{\mathcal { O }}_{\left[z_{[i: n]} ; \theta, \beta\right]}\right\}^{-b}-1\right)^{-2}\right]\right\}^{a}-v_{(i, n)}^{[2]}\right] \delta_{\theta}\left(z_{[i: n]}, \underline{\boldsymbol{\Phi}}\right),
\end{aligned}
$$

$$
0=\sum_{i=1}^{n}\left[\left\{1-\exp \left[-\left(\left\{1-\boldsymbol{\mathcal { O }}_{\left[z_{[i: n]} ; \theta, \beta\right]}\right\}^{-b}-1\right)^{-2}\right]\right\}^{a}-v_{(i, n)}^{[2]}\right] \delta_{\beta}\left(z_{[i: n]}, \underline{\Phi}\right),
$$

\subsection{The WLSE method}

The WLSE are obtained by minimizing the function $\operatorname{WLSE}(\underline{\boldsymbol{\Phi}})$ WRT $a, b, \theta$ and $\beta$ from 
where

$$
\operatorname{WLSE}(\underline{\boldsymbol{\Phi}})=\sum_{i=1}^{n} v_{(i, n)}^{[3]}\left[F_{\underline{\Phi}}\left(z_{[i: n]}\right)-v_{(i, n)}^{[2]}\right]^{2}
$$

The WLSEs are obtained by solving

$$
v_{(i, n)}^{[3]}=\left[(1+n)^{2}(2+n)\right] /[i(1+n-i)] .
$$

and

$$
\begin{aligned}
& 0=\sum_{i=1}^{n} v_{(i, n)}^{[3]}\left[\left\{1-\exp \left[-\left(\left\{1-\boldsymbol{\mathcal { O }}_{\left[z_{[i: n]} ; \theta, \beta\right]}\right\}^{-b}-1\right)^{-2}\right]\right\}^{a}-v_{(i, n)}^{[2]]}\right] \delta_{a}\left(z_{[i: n]}, \underline{\boldsymbol{\Phi}}\right), \\
& 0=\sum_{i=1}^{n} v_{(i, n)}^{[3]}\left[\left\{1-\exp \left[-\left(\left\{1-\boldsymbol{\mathcal { O }}_{\left[z_{[i: n]} ; \theta, \beta\right]}\right\}^{-b}-1\right)^{-2}\right]\right\}^{a}-v_{(i, n)}^{[2]}\right] \delta_{b}\left(z_{[i: n]}, \underline{\boldsymbol{\Phi}}\right), \\
& 0=\sum_{i=1}^{n} v_{(i, n)}^{[3]}\left[\left\{1-\exp \left[-\left(\left\{1-\boldsymbol{\mathcal { O }}_{\left[z_{[i: n]} ; \theta, \beta\right]}\right\}^{-b}-1\right)^{-2}\right]\right\}^{a}-v_{(i, n)}^{[2]}\right] \delta_{\theta}\left(z_{[i: n]}, \underline{\boldsymbol{\Phi}}\right),
\end{aligned}
$$

$$
0=\sum_{i=1}^{n} v_{(i, n)}^{[3]}\left[\left\{1-\exp \left[-\left(\left\{1-\boldsymbol{\mathcal { O }}_{\left[z_{[i: n]} ; \theta, \beta\right]}\right\}^{-b}-1\right)^{-2}\right]\right\}^{a}-v_{(i, n)}^{[2]}\right] \delta_{\beta}\left(z_{[i: n]}, \underline{\boldsymbol{\Phi}}\right)
$$

\subsection{The ADE method}

The ADEs are obtained by minimizing the function

$$
A D E(\underline{\boldsymbol{\Phi}})=-n-n^{-1} \sum_{i=1}^{n}(2 i-1)\left\{\log F_{\underline{\Phi}}\left(z_{[i: n]}\right)+\log \left[1-F_{\underline{\Phi}}\left(w_{[-i+1+n: n]}\right)\right]\right\} .
$$

The parameter estimates follow by solving the nonlinear equations

$$
\frac{\partial}{\partial a}[A D E(\underline{\boldsymbol{\Phi}})]=0, \frac{\partial}{\partial b}[A D E(\underline{\boldsymbol{\Phi}})]=0, \frac{\partial}{\partial \theta}[A D E(\underline{\boldsymbol{\Phi}})]=0 \text { and } \frac{\partial}{\partial \beta}[A D E(\underline{\boldsymbol{\Phi}})]=0 .
$$

\subsection{The RTADE method}

The RTADEs are obtained by minimizing

$$
\operatorname{RTADE}(\underline{\boldsymbol{\Phi}})=\frac{1}{2} n-2 \sum_{i=1}^{n} F_{\underline{\Phi}}\left(z_{[i: n]}\right)-\frac{1}{n} \sum_{i=1}^{n}(2 i-1) \log \left[1-F_{\underline{\Phi}}\left(z_{[-i+1+n: n]}\right)\right] .
$$

The parameter estimates follow by solving the nonlinear equations

$$
\frac{\partial}{\partial a}[R T A D E(\underline{\boldsymbol{\Phi}})]=0, \frac{\partial}{\partial b}[R T A D E(\underline{\boldsymbol{\Phi}})]=0, \frac{\partial}{\partial \theta}[R T A D E(\underline{\boldsymbol{\Phi}})]=0 \text { and } \frac{\partial}{\partial \beta}[R T A D E(\underline{\boldsymbol{\Phi}})]=0 .
$$

\subsection{The LTADE method}

The R.T.ADEs are obtained by minimizing

$$
\operatorname{LTADE}(\underline{\boldsymbol{\Phi}})=-\frac{3}{2} n+2 \sum_{i=1}^{n} F_{\underline{\Phi}}\left(z_{[i: n]}\right)-\frac{1}{n} \sum_{i=1}^{n}(2 i-1) \log F_{\underline{\Phi}}\left(z_{[i: n]}\right) .
$$

The parameter estimates follow by solving the nonlinear equations

$$
\frac{\partial}{\partial a}[\operatorname{LTADE}(\underline{\boldsymbol{\Phi}})]=0, \frac{\partial}{\partial b}[\operatorname{LTADE}(\underline{\boldsymbol{\Phi}})]=0, \frac{\partial}{\partial \theta}[\operatorname{LTADE}(\underline{\boldsymbol{\Phi}})]=0, \frac{\partial}{\partial \beta}[\operatorname{LTADE}(\underline{\boldsymbol{\Phi}})]=0 .
$$

\section{Simulation study for comparing non-Bayesian estimation methods}

A numerical simulation is performed in to compare the classical estimation methods. The simulation study is based on $\mathrm{N}=1000$ generated data sets from the BXGNH version where $n=50,100,150$ and 300 and

$\begin{array}{ccccc} & a & b & \theta & \beta \\ \text { I } & 0.8 & 0.8 & 0.8 & 0.8 \\ \text { II } & 1.2 & 0.5 & 1.5 & 0.6 \\ \text { III } & 3.0 & 0.15 & 0.75 & 0.3\end{array}$


The estimates are compared in terms of:

$1-\operatorname{Bias}\left(\operatorname{BIAS}_{(\underline{\boldsymbol{\Phi}})}\right)$;

2-Root mean-standard error $\left(\operatorname{RMSE}_{(\underline{\underline{\Phi}})}\right)$;

3-The mean of the absolute difference between the theoretical and the estimates $\left(D_{(\mathrm{abs})}\right)$ and

4-the maximum absolute difference between the true parameters and estimates $\left(D_{\max }\right)$.

Table 3: Simulation results for parameters $\mathrm{a}=0.8, \mathrm{~b}=0.8, \theta=0.8$ and $\beta=0.8$.

\begin{tabular}{|c|c|c|c|c|c|c|c|c|c|c|c|}
\hline & & \multicolumn{4}{|c|}{ BIAS } & \multicolumn{4}{|c|}{ RMSE } & \multicolumn{2}{|c|}{$\mathrm{D}$} \\
\hline & $\mathrm{n}$ & $\mathrm{a}$ & $\mathrm{b}$ & $\theta$ & $\beta$ & $\mathrm{a}$ & $\mathrm{b}$ & $\theta$ & $\beta$ & D.abs & D.max \\
\hline MLE & 50 & 0.01927 & 0.00141 & 0.003614 & 0.00431 & 0.121200 & 0.045730 & 0.03854 & 0.052940 & 0.003070 & 0.00558 \\
\hline CVM & & 0.019773 & 0.002873 & 0.001705 & 0.002375 & 0.139617 & 0.051192 & 0.051883 & 0.067038 & 0.005678 & 0.00902 \\
\hline OLS & & 0.048233 & 0.01366 & -0.008955 & -0.011326 & 0.151655 & 0.053358 & 0.051404 & 0.06624 & 0.039810 & 0.05491 \\
\hline WLS & & 0.073721 & 0.017441 & -0.007943 & -0.011474 & 0.151569 & 0.050682 & 0.041513 & 0.056031 & 0.049748 & 0.068395 \\
\hline $\mathrm{ADE}$ & & 0.014963 & 0.003444 & -0.000871 & -0.000471 & 0.12924 & 0.048678 & 0.045292 & 0.060119 & 0.008379 & 0.011559 \\
\hline RTADE & & 0.028109 & 0.00486 & -0.001626 & -0.001724 & 0.156276 & 0.047911 & 0.041809 & 0.056027 & 0.014888 & 0.020531 \\
\hline LEADE & & 0.010471 & 0.001115 & 0.004229 & 0.005688 & 0.123644 & 0.054829 & 0.060846 & 0.077492 & 0.003175 & 0.006413 \\
\hline MLE & 150 & 0.00598 & -0.00064 & 0.00254 & 0.00317 & 0.06448 & 0.02654 & 0.02289 & 0.03119 & 0.00263 & 0.00501 \\
\hline CVM & & 0.005506 & 0.000332 & 0.001349 & 0.001728 & 0.074428 & 0.028811 & 0.028952 & 0.037408 & 0.000937 & 0.00158 \\
\hline OLS & & 0.014632 & 0.003883 & -0.002185 & -0.002821 & 0.076629 & 0.029161 & 0.028779 & 0.037158 & 0.011111 & 0.015221 \\
\hline WLS & & 0.030762 & 0.005396 & -0.000631 & -0.001385 & 0.076692 & 0.028622 & 0.023991 & 0.03264 & 0.015204 & 0.021054 \\
\hline ADE & & 0.003843 & 0.000396 & 0.000645 & 0.000979 & 0.069724 & 0.027412 & 0.025692 & 0.033988 & 0.000649 & 0.001203 \\
\hline RTADE & & 0.006492 & 0.00045 & 0.000631 & 0.000932 & 0.083313 & 0.027194 & 0.023998 & 0.032076 & 0.001385 & 0.002427 \\
\hline LEADE & & 0.003309 & 0.000216 & 0.001678 & 0.002143 & 0.0663 & 0.030533 & 0.033224 & 0.042309 & 0.001463 & 0.002856 \\
\hline MLE & 300 & 0.00255 & 0.00016 & 0.00044 & 0.00056 & 0.04770 & 0.01801 & 0.01461 & 0.02010 & 0.00041 & 0.00076 \\
\hline CVM & & 0.001667 & -0.000047 & 0.000732 & 0.000994 & 0.052991 & 0.020591 & 0.020452 & 0.026504 & 0.000715 & 0.001386 \\
\hline OLS & & 0.006196 & 0.001732 & -0.001037 & -0.001285 & 0.053669 & 0.020694 & 0.020384 & 0.026401 & 0.004907 & 0.006743 \\
\hline WLS & & 0.020055 & 0.003554 & -0.000603 & -0.001124 & 0.053842 & 0.019257 & 0.015422 & 0.021208 & 0.010236 & 0.01416 \\
\hline ADE & & 0.00126 & 0.000177 & 0.000117 & 0.000302 & 0.050258 & 0.0195 & 0.017937 & 0.023847 & 0.000278 & 0.000491 \\
\hline RTADE & & 0.003154 & 0.00053 & -0.000158 & -0.0001 & 0.057986 & 0.018844 & 0.016364 & 0.021962 & 0.001596 & 0.002206 \\
\hline LEADE & & 0.00061 & -0.000381 & 0.001285 & 0.001703 & 0.048742 & 0.022386 & 0.024027 & 0.030716 & 0.002121 & 0.003344 \\
\hline MLE & 500 & 0.00425 & 0.00048 & 0.00042 & 0.00042 & 0.03515 & 0.01401 & 0.01164 & 0.01599 & 0.00115 & 0.00185 \\
\hline CVM & & 0.003883 & 0.000914 & -0.000359 & -0.000485 & 0.040727 & 0.015783 & 0.015581 & 0.020152 & 0.002525 & 0.003455 \\
\hline OLS & & 0.006601 & 0.001979 & -0.001416 & -0.001847 & 0.041235 & 0.015895 & 0.015605 & 0.02018 & 0.005875 & 0.008093 \\
\hline WLS & & 0.016201 & 0.002558 & -0.000077 & -0.000423 & 0.040173 & 0.01472 & 0.012045 & 0.016516 & 0.007422 & 0.010369 \\
\hline $\mathrm{ADE}$ & & 0.003464 & 0.000913 & -0.000466 & -0.000605 & 0.038306 & 0.01498 & 0.013882 & 0.018365 & 0.002548 & 0.003488 \\
\hline RTADE & & 0.004139 & 0.000759 & -0.000314 & -0.00041 & 0.045107 & 0.014753 & 0.0129 & 0.017251 & 0.002397 & 0.003285 \\
\hline LEADE & & 0.00338 & 0.001089 & -0.000519 & -0.000679 & 0.036664 & 0.016836 & 0.017913 & 0.022844 & 0.002749 & 0.003773 \\
\hline
\end{tabular}

Table 4: Simulation results for parameters $\mathrm{a}=1.2, \mathrm{~b}=0.5, \theta=1.5$ and $\beta=0.6$.

\begin{tabular}{|c|c|c|c|c|c|c|c|c|c|c|c|}
\hline & & \multicolumn{4}{|c|}{ BIAS } & \multicolumn{4}{|c|}{ RMSE } & \multicolumn{2}{|c|}{$\mathrm{D}$} \\
\hline & $\mathrm{n}$ & $\mathrm{a}$ & $\mathrm{b}$ & $\theta$ & $\beta$ & $\mathrm{a}$ & $\mathrm{b}$ & $\theta$ & $\beta$ & D.abs & D.max \\
\hline MLE & 50 & 0.02542 & 0.00027 & 0.00820 & 0.00351 & 0.18033 & 0.02384 & 0.08545 & 0.03857 & 0.00260 & 0.00426 \\
\hline CVM & & 0.033587 & 0.001691 & 0.002964 & 0.001676 & 0.215409 & 0.027513 & 0.092424 & 0.051911 & 0.006838 & 0.010564 \\
\hline OLS & & 0.058898 & 0.005299 & -0.012815 & -0.005605 & 0.20657 & 0.025645 & 0.105434 & 0.046216 & 0.029892 & 0.041151 \\
\hline WLS & & 0.110834 & 0.009003 & -0.017515 & -0.008081 & 0.235249 & 0.027897 & 0.096978 & 0.043477 & 0.049831 & 0.068825 \\
\hline $\mathrm{ADE}$ & & 0.009573 & -0.000335 & 0.007204 & 0.003407 & 0.190087 & 0.025749 & 0.103057 & 0.045831 & 0.003967 & 0.007034 \\
\hline RTADE & & 0.033066 & 0.001659 & -0.000885 & -0.000243 & 0.228697 & 0.024646 & 0.090906 & 0.040585 & 0.009923 & 0.013965 \\
\hline LEADE & & 0.011595 & -0.000361 & 0.01354 & 0.005955 & 0.183055 & 0.028314 & 0.130824 & 0.057103 & 0.00793 & 0.013301 \\
\hline MLE & 150 & 0.01056 & 0.00088 & -0.00018 & -0.00015 & 0.09835 & 0.01411 & 0.05049 & 0.02277 & 0.00376 & 0.00526 \\
\hline CVM & & 0.004158 & -0.000221 & 0.00312 & 0.001767 & 0.111601 & 0.014929 & 0.049715 & 0.027921 & 0.002317 & 0.003934 \\
\hline OLS & & 0.019959 & 0.001825 & -0.004429 & -0.001935 & 0.112852 & 0.0149 & 0.061417 & 0.026969 & 0.01022 & 0.014099 \\
\hline WLS & & 0.050715 & 0.003323 & -0.003336 & -0.001695 & 0.120708 & 0.015296 & 0.053194 & 0.023958 & 0.018276 & 0.025311 \\
\hline $\mathrm{ADE}$ & & 0.005882 & 0.000206 & 0.001471 & 0.000683 & 0.103874 & 0.014278 & 0.056608 & 0.025096 & 0.000638 & 0.001197 \\
\hline RTADE & & 0.017447 & 0.001345 & -0.003102 & -0.001333 & 0.127814 & 0.014553 & 0.053835 & 0.023998 & 0.007948 & 0.010969 \\
\hline LEADE & & 0.006487 & 0.000504 & 0.001003 & 0.000471 & 0.101028 & 0.01599 & 0.071518 & 0.031211 & 0.001387 & 0.002168 \\
\hline MLE & 300 & 0.00328 & -0.00015 & 0.00206 & 0.00090 & 0.06820 & 0.00971 & 0.03444 & 0.01554 & 0.00106 & 0.00193 \\
\hline CVM & & 0.004645 & 0.000108 & 0.001134 & 0.00063 & 0.081853 & 0.011003 & 0.03647 & 0.020491 & 0.000464 & 0.000771 \\
\hline OLS & & 0.0108 & 0.001073 & -0.00294 & -0.001279 & 0.081307 & 0.010844 & 0.04454 & 0.01957 & 0.006048 & 0.008368 \\
\hline WLS & & 0.034075 & 0.002166 & -0.002044 & -0.00106 & 0.079443 & 0.010257 & 0.035653 & 0.016083 & 0.012052 & 0.016728 \\
\hline ADE & & 0.001358 & -0.00008 & 0.001229 & 0.000579 & 0.075584 & 0.010312 & 0.040321 & 0.017908 & 0.000764 & 0.001296 \\
\hline RTADE & & 0.003544 & 0.000028 & 0.000788 & 0.000378 & 0.088211 & 0.010079 & 0.037194 & 0.016602 & 0.000343 & 0.000629 \\
\hline LEADE & & 0.000745 & -0.000242 & 0.00304 & 0.001332 & 0.072173 & 0.011512 & 0.051499 & 0.022474 & 0.002415 & 0.003703 \\
\hline MLE & 500 & 0.00272 & 0.00001 & 0.00107 & 0.00046 & 0.05492 & 0.00790 & 0.02795 & 0.01262 & 0.00035 & 0.00067 \\
\hline CVM & & 0.002818 & 0.000177 & 0.000077 & 0.000051 & 0.059576 & 0.008093 & 0.026893 & 0.015106 & 0.000789 & 0.001131 \\
\hline OLS & & 0.010912 & 0.001163 & -0.003655 & -0.001612 & 0.062959 & 0.008413 & 0.034483 & 0.01514 & 0.006759 & 0.009363 \\
\hline WLS & & 0.021556 & 0.001203 & -0.000551 & -0.000325 & 0.060518 & 0.007751 & 0.026788 & 0.012098 & 0.006816 & 0.009547 \\
\hline $\mathrm{ADE}$ & & 0.002061 & 0.000093 & 0.000336 & 0.000163 & 0.060022 & 0.008229 & 0.03225 & 0.014306 & 0.000324 & 0.000553 \\
\hline RTADE & & 0.00348 & 0.000112 & 0.000282 & 0.000136 & 0.070455 & 0.008125 & 0.03003 & 0.013396 & 0.000686 & 0.001058 \\
\hline LEADE & & 0.001654 & 0.000053 & 0.000989 & 0.000432 & 0.057004 & 0.009069 & 0.040275 & 0.017569 & 0.000339 & 0.000674 \\
\hline
\end{tabular}


Table 5: Simulation results for parameters $a=3, b=0.15, \theta=0.75$ and $\beta=0.3$.

\begin{tabular}{|c|c|c|c|c|c|c|c|c|c|c|c|}
\hline & \multirow[b]{2}{*}{$\mathrm{n}$} & \multicolumn{4}{|c|}{ BIAS } & \multicolumn{4}{|c|}{ RMSE } & \multicolumn{2}{|c|}{ D } \\
\hline & & $\mathrm{a}$ & $\mathrm{b}$ & $\theta$ & $\beta$ & $\mathrm{a}$ & $\mathrm{b}$ & $\theta$ & $\beta$ & D.abs & D.max \\
\hline MLE & 50 & 0.06355 & 0.00010 & 0.00844 & 0.00339 & 0.45083 & 0.00510 & 0.08887 & 0.03668 & 0.00227 & 0.00397 \\
\hline CVM & & 0.038512 & -0.000672 & 0.000662 & 0.000443 & 0.49847 & 0.053671 & 0.013399 & 0.008599 & 0.001737 & 0.00325 \\
\hline OLS & & 0.159551 & 0.001102 & -0.008763 & -0.00356 & 0.581273 & 0.005813 & 0.105462 & 0.042983 & 0.028699 & 0.039982 \\
\hline WLS & & 0.243182 & 0.001705 & -0.015295 & -0.006342 & 0.545509 & 0.005618 & 0.093092 & 0.038274 & 0.04535 & 0.063472 \\
\hline $\mathrm{ADE}$ & & 0.031012 & 0.00002 & 0.007493 & 0.00314 & 0.472442 & 0.005247 & 0.097703 & 0.04003 & 0.003418 & 0.005957 \\
\hline RTADE & & 0.087423 & 0.000285 & 0.000662 & 0.001807 & 0.605563 & 0.005466 & 0.018608 & 0.042221 & 0.006365 & 0.009867 \\
\hline LEADE & & 0.034739 & -0.00001 & 0.013571 & 0.005525 & 0.465023 & 0.005807 & 0.122543 & 0.049819 & 0.008028 & 0.012719 \\
\hline MLE & 150 & 0.02638 & 0.00019 & -0.00012 & -0.00008 & 0.24588 & 0.00299 & 0.05178 & 0.02135 & 0.00377 & 0.00535 \\
\hline CVM & & -0.002383 & -0.001691 & 0.000567 & 0.000374 & 0.282469 & 0.031234 & 0.00778 & 0.004988 & 0.004988 & 0.007143 \\
\hline OLS & & 0.045269 & 0.00034 & -0.002995 & -0.001213 & 0.289525 & 0.00314 & 0.058303 & 0.023769 & 0.008752 & 0.012235 \\
\hline WLS & & 0.121141 & 0.000871 & -0.008549 & -0.003556 & 0.288142 & 0.003184 & 0.053122 & 0.021889 & 0.023629 & 0.033025 \\
\hline ADE & & 0.003751 & -0.000053 & 0.003461 & 0.001439 & 0.262112 & 0.002995 & 0.055117 & 0.022558 & 0.00289 & 0.004289 \\
\hline RTADE & & 0.017853 & -0.000001 & 0.000535 & 0.001273 & 0.303672 & 0.002965 & 0.010274 & 0.023250 & 0.000931 & 0.001767 \\
\hline LEADE & & 0.005791 & -0.000056 & 0.004847 & 0.001971 & 0.239979 & 0.003065 & 0.062176 & 0.046817 & 0.019033 & 0.002221 \\
\hline MLE & 300 & 0.00818 & -0.00002 & 0.00213 & 0.000870 & 0.17051 & 0.00207 & 0.03556 & 0.01468 & 0.00121 & 0.00200 \\
\hline CVM & & 0.015518 & 0.00083 & -0.000114 & -0.000071 & 0.204527 & 0.022424 & 0.005547 & 0.003557 & 0.002616 & 0.003672 \\
\hline OLS & & 0.027204 & 0.000198 & -0.001733 & -0.000706 & 0.206089 & 0.002271 & 0.04234 & 0.017262 & 0.005176 & 0.007219 \\
\hline WLS & & 0.076367 & 0.000427 & -0.002307 & -0.000997 & 0.200766 & 0.002259 & 0.037994 & 0.015682 & 0.011493 & 0.01613 \\
\hline $\mathrm{ADE}$ & & 0.015651 & 0.000097 & -0.000119 & -0.000044 & 0.192103 & 0.002183 & 0.039849 & 0.01631 & 0.002136 & 0.003021 \\
\hline RTADE & & 0.013691 & 0.000073 & -0.000026 & 0.000031 & 0.222764 & 0.002197 & 0.007562 & 0.017126 & 0.001721 & 0.002449 \\
\hline LEADE & & 0.001272 & -0.000048 & 0.002922 & 0.001192 & 0.181657 & 0.002323 & 0.046817 & 0.000037 & 0.002601 & 0.003812 \\
\hline MLE & 500 & 0.00678 & 0.000010 & 0.001030 & 0.000410 & 0.13733 & 0.001680 & 0.028820 & 0.011890 & 0.000290 & 0.00056 \\
\hline CVM & & 0.00346 & -0.000115 & 0.000079 & 0.000053 & 0.154813 & 0.017218 & 0.004276 & 0.002742 & 0.000296 & 0.000505 \\
\hline OLS & & 0.017013 & 0.000147 & -0.001804 & -0.000732 & 0.151693 & 0.001679 & 0.031298 & 0.012761 & 0.003987 & 0.005564 \\
\hline WLS & & 0.052412 & 0.000269 & -0.001042 & -0.000463 & 0.153261 & 0.001736 & 0.029212 & 0.012056 & 0.007280 & 0.010256 \\
\hline $\mathrm{ADE}$ & & 0.010287 & 0.000089 & -0.000905 & -0.000362 & 0.145138 & 0.001642 & 0.029617 & 0.012125 & 0.002260 & 0.003153 \\
\hline RTADE & & 0.006039 & 0.000023 & 0.000051 & 0.000162 & 0.169091 & 0.001665 & 0.005709 & 0.012923 & 0.000430 & 0.000678 \\
\hline LEADE & & -0.002878 & -0.000065 & 0.002301 & 0.00094 & 0.137424 & 0.001763 & 0.035203 & 0.014312 & 0.002578 & 0.003684 \\
\hline
\end{tabular}

From Tables 3, 4 and 5 we note that:

1-The BIAS $(\underline{\boldsymbol{\Phi}})$ tend to zero when $\mathrm{n}$ increases which means that all estimators are non-biased.

2-The RMSE $(\underline{\boldsymbol{\Phi}})$ reduces and tend to zero when $\mathrm{n}$ increases which means incidence of consistency property.

\section{Comparing competitive model under the uncensored MLE method}

In this section, we present an application based on the real data set to show the flexibility of the BXGNH distribution. First, we compare BXGNH with the RNH, the odd Lindley NH distribution (OLNH) (Yousof et al., (2017)), Proportional reversed hazard rate (PRHRNH) (new), exponentiated Weibull NH (New), the Gamma-NH (GaNH) (Ortega et al., (2015)), Marshall-Olkin NH (MONH) (Lemonte et al., (2016)), generalized NH (GNH) (Lemonte (2013)), beta-NH (BNH ) (Dias et al., (2018)), the standard NH distributions. Other useful extension of the NH model such as the Topp-Leone NH distribution (Yousof and Korkmaz (2017)) and extended GNH model (Alizadeh et al., (2018)). The model selection is applied using the estimated log-likelihood, Kolmogorov-Smirnov (K-S) statistics, Akaike information criterion $\left(\mathcal{W}_{1}\right)$, Consistent Akaike information criteria $\left(\mathcal{W}_{2}\right)$, Bayesian information criterion $\left(\mathcal{W}_{3}\right)$, and Hannan-Quinn information criterion $\left(\mathcal{W}_{4}\right)$. The total time on test (TTT) plot is given for the used data set. Finally, we present the estimated PDF, estimated CDF, estimated HRF, P-P and Kaplan-Meier survival plots of the BXGNH for the used data set (the exceedances of flood peaks data). The used data corresponds to the exceedances of flood peaks (in $\mathrm{m}^{3} / \mathrm{s}$ ) of the Wheaton River near Carcross in Yukon Territory, Canada. These data consist of 72 exceedances for the years 1958--1984, rounded to one decimal place (see Choulakian and Stephens (2001)). This data also has been applied by Lemonte (2013) for the GNH distribution. In the applications, the information about the hazard shape can help in selecting a particular model. For this aim, an important tool called the TTT plot (see Aarset (1987)) is useful. The TTT plot (left) and the box plot (right) for the exceedances of flood peaks data are given in Figure 3 . Due to Figure 3, the HRF is a bathtub-shaped (U-HRF). The box plot refers that, we have only an extreme value. Figure 4 gives the quantile-quantile (Q-Q) plot (left) and Kernel density estimation (KDE) (right) for the exceedances of flood peaks data. All results of this application are listed in Table 6 and Table 7 . These results in Table 7 show that the BXGNH distribution has the best fits where Log-like. $=-249.017, \mathcal{W}_{1}=506.034, \mathcal{W}_{2}=506.63, \mathcal{W}_{3}=513.14, \mathcal{W}_{4}=509.6$, $\mathrm{K}-\mathrm{S}=0.0980$ and also has the biggest estimated log-likelihood $(-249.0169)$ and $\mathrm{p}$-value $(\mathbf{P} v=0.50)$. Thus, BXGNH could be chosen as the best NH model. Finally, we plot estimated functions for the density, CDF, P-P, Kaplan-Meier survival plots of the BXGNH for the exceedances of flood peaks data in Figure 5. For other useful real data see Merovci et al. (2017), Altun et al. (2018a-e), Bhatti et al. (2018, 2019), Yadav et al. (2019), Gad et al. (2019), Korkmaz 
et al. (2019), Alizadeh et al. (2020), Merovci et al. (2020), Bhatti et al. (2020, 2021), Altun et al. (2021) and ElMorshedy et al. (2021).

Table 6: Estimates of the competitive models.

\begin{tabular}{|c|c|c|c|c|}
\hline Model & & Estim & (SDs) & \\
\hline $\mathrm{NH}(\theta, \beta)$ & 0.8412 & 0.1094 & & \\
\hline & $(0.259)$ & $(0.059)$ & & \\
\hline $\operatorname{RNH}(\theta, \beta)$ & 0.125 & 6.28 & & \\
\hline & $(0.012)$ & (2.919) & & \\
\hline $\operatorname{RGNH}(b, \theta)$ & 0.6038 & 0.1311 & & \\
\hline & $(0.073)$ & $(0.0163)$ & & \\
\hline $\operatorname{RGNH}(b, \theta, \beta)$ & 2.4549 & 0.1126 & 213.97 & \\
\hline & $(2.226)$ & $(0.0204)$ & $(893.1)$ & \\
\hline OLNH $(a, \theta, \beta)$ & 0.7293 & 0.2519 & 1.8065 & \\
\hline & $(0.606)$ & $(0.052)$ & $(3.355)$ & \\
\hline $\operatorname{PRHRNH}(a, \theta, \beta)$ & 0.364 & 1.714 & 0.031 & \\
\hline & $(0.068)$ & (1.191) & $(0.031)$ & \\
\hline $\operatorname{GaNH}(\mathrm{a}, \theta, \beta)$ & 0.7286 & 1.9299 & 0.0242 & \\
\hline & $(0.1385)$ & $(1.7591)$ & $(0.031)$ & \\
\hline $\operatorname{MONH}(\mathrm{a}, \theta, \beta)$ & 23.77 & 0.0011 & 0.2660 & \\
\hline $\mathrm{GNH}(\mathrm{a}, \theta, \beta)$ & 0.7289 & 1.7126 & 0.0309 & \\
\hline & $(0.1404)$ & $(1.2607)$ & $(0.033)$ & \\
\hline $\mathrm{BNH}(\mathrm{a}, \mathrm{b}, \theta, \beta)$ & 0.8381 & 316.0285 & 0.6396 & 0.0003 \\
\hline & $(0.1215)$ & $(4.2194)$ & $(0.823)$ & $(0.0004)$ \\
\hline $\operatorname{EWNH}(a, b, \theta, \beta)$ & 2.7591 & 0.3989 & 0.4732 & 0.6129 \\
\hline & $(1.742)$ & $(0.167)$ & $(0.158)$ & $(0.959)$ \\
\hline $\operatorname{BXGNH}(a, b, \theta, \beta)$ & 0.2095 & 4.4422 & 0.2422 & 2.7248 \\
\hline & $(0.0347)$ & $(0.7764)$ & 90.0129) & $(0.8498)$ \\
\hline
\end{tabular}

Table 7: Statistics of the competitive models fitted to the Choulakian and Stephens data.

\begin{tabular}{lcccccc}
\hline Model & Log-like. & $\mathcal{W}_{1}$ & $\mathcal{W}_{2}$ & $\mathcal{W}_{3}$ & $\mathcal{W}_{4}$ & K-S $(\mathbf{P} v)$ \\
\hline BXGNH & $\mathbf{- 2 4 9 . 0 1 7}$ & $\mathbf{5 0 6 . 0 3 4}$ & $\mathbf{5 0 6 . 6 3}$ & $\mathbf{5 1 3 . 1 4}$ & $\mathbf{5 0 9 . 6 3}$ & $\mathbf{0 . 0 9 8 0 1 0}(\mathbf{0 . 5 0 2})$ \\
RGNH & -582.723 & 1171.45 & 1171.8 & 1178.3 & 1174.2 & $0.09616(0.519)$ \\
RGNH & -584.004 & 1172.01 & 1172.2 & 1176.6 & 1173.8 & $0.10227(0.439)$ \\
RNH & -251.722 & 507.44 & 507.62 & 513.99 & 509.73 & $0.10629(0.390)$ \\
NH & -251.987 & 507.973 & 508.15 & 515.53 & 509.79 & $0.12444(0.215)$ \\
OLNH & -250.589 & 507.181 & 507.53 & 514.01 & 509.92 & $0.10092(0.457)$ \\
PRHRNH & -300.833 & 607.662 & 608.02 & 614.49 & 610.38 & $0.24985(0.001)$ \\
GaNH & -250.917 & 507.834 & 508.19 & 514.66 & 510.55 & $0.10651(0.388)$ \\
MONH & -251.087 & 508.175 & 508.53 & 515.01 & 510.89 & $0.10744(0.377)$ \\
EWNH & -250.032 & 508.064 & 508.66 & 517.17 & 511.69 & $0.09743(0.510)$ \\
GNH & -250.925 & 507.849 & 508.20 & 514.68 & 510.57 & $0.10672(0.386)$ \\
BNH & -251.356 & 510.713 & 511.31 & 519.82 & 514.34 & $0.10444(0.413)$ \\
& & & & & & \\
\hline
\end{tabular}



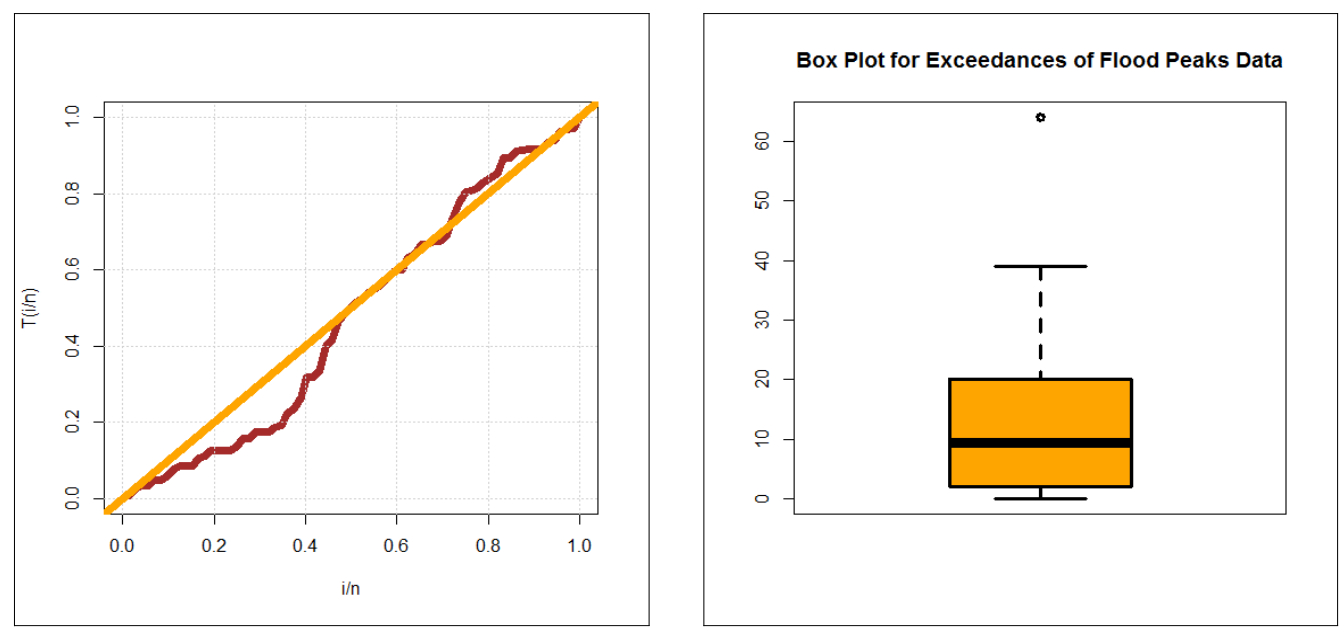

Figure 3: TTT plot and box plot for the exceedances of flood peaks data.
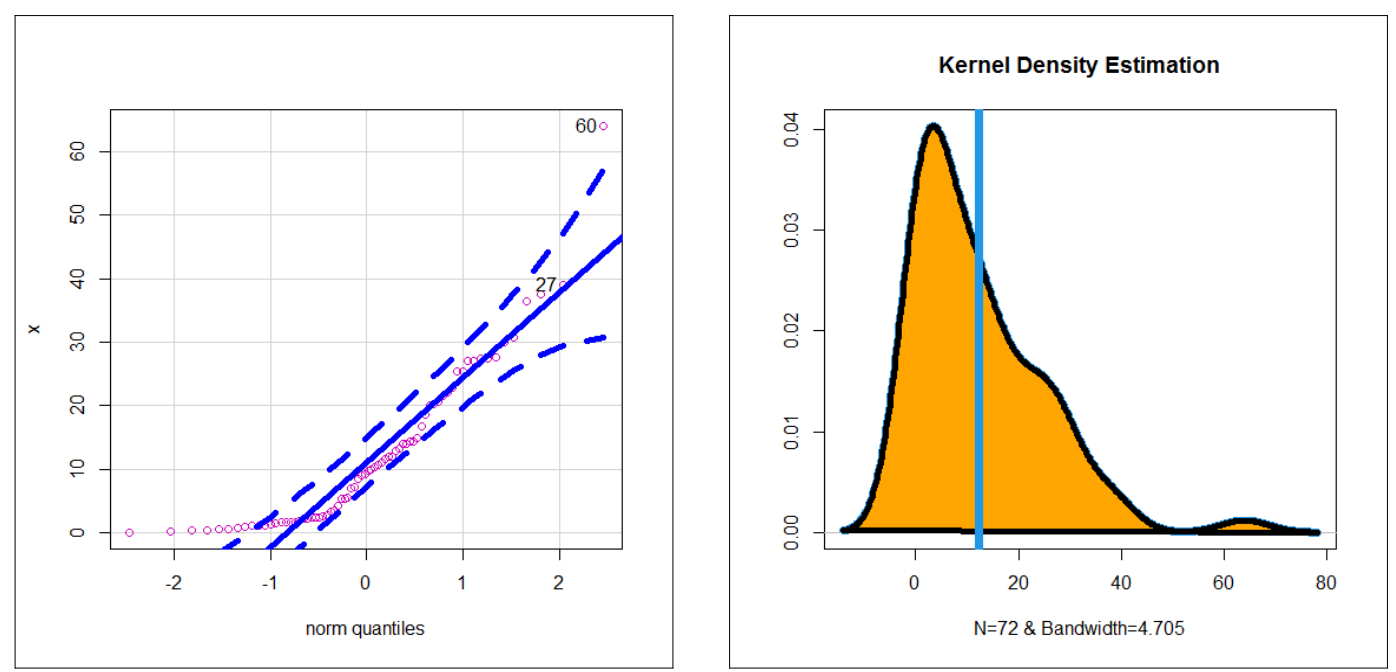

Figure 4: Q-Q plot and KDE for the exceedances of flood peaks data. 

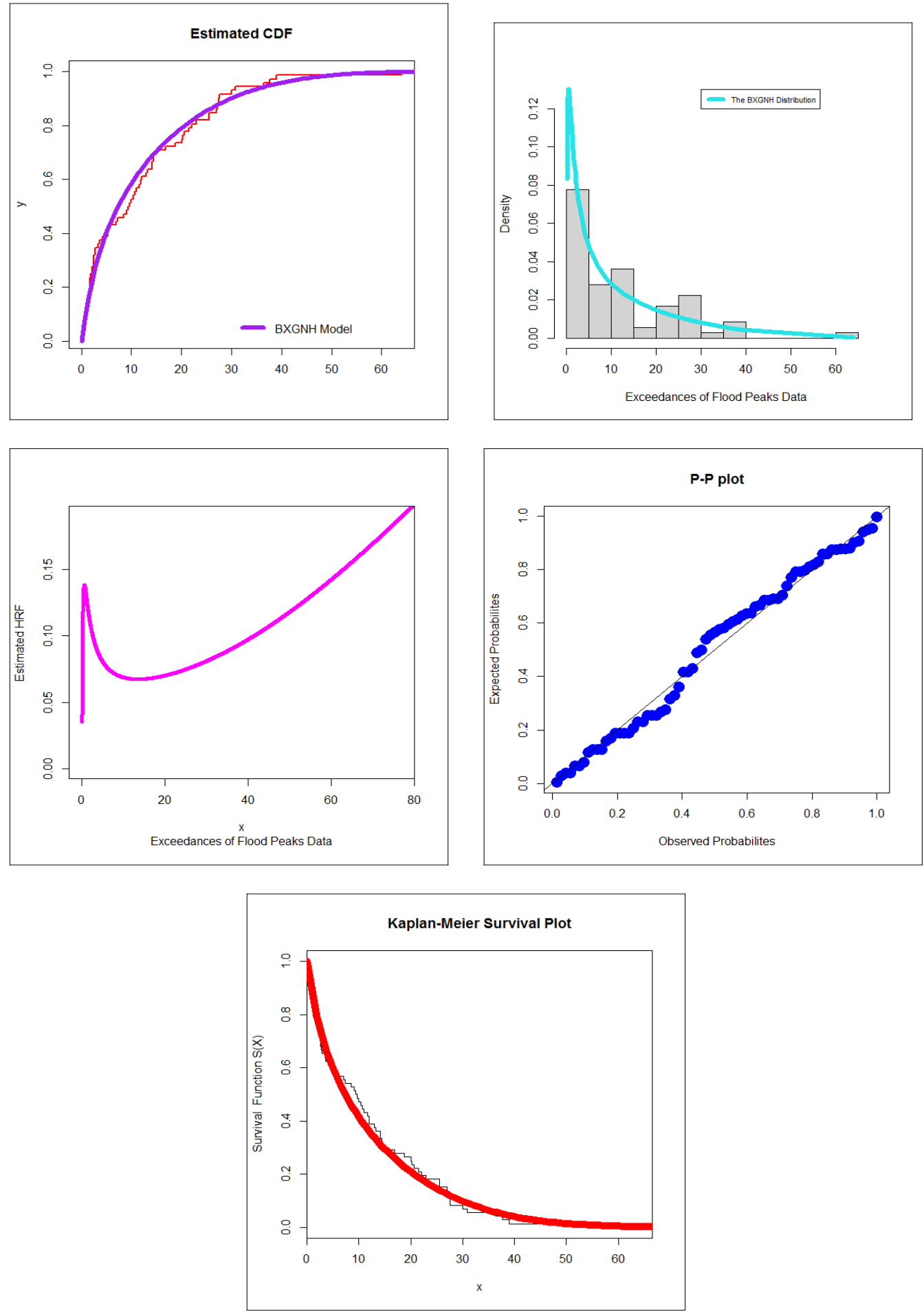

Figure 5: Estimated CDF, PDF, HRF, P-P, Kaplan-Meier survival plots of the BXGNH for the exceedances of flood peaks data. 


\section{Maximum likelihood estimation in censored data case}

Suppose that $Z_{1}, Z_{2}, \ldots \ldots, Z_{n}$ is a random sample with right censoring from BXGNH $(\underline{\Phi})$ distribution. The observed data

$$
\left.z_{i}\right|_{(i=1,2, . ., n)}=\min \left(Z_{i}, C_{i}\right)
$$

are the minimum of the survival time $Z_{i}$ and censoring time $C_{i}$ for each subject in the sample. So, $z_{i}$ can be written in the form $\left(z_{i}, \boldsymbol{\nabla}_{i}\right)_{i=1, \ldots, n}$ where $\boldsymbol{\nabla}_{i}=1$ if $Z_{i}$ is the moment of failure (complete observation) and $\boldsymbol{\nabla}_{i}=0$ if $Z_{i}$ is the moment of censoring. The right censoring is assumed to be non-informative, so the expression of the likelihood function is

$$
\left.l(z, \underline{\Phi})=\left.\Pi_{i=1}^{n} f_{\underline{\Phi}}\left(z_{i}\right)^{\nabla_{i}} S_{\underline{\Phi}}\left(z_{i}\right)^{1-\nabla_{i}}\right|_{\left(\nabla_{i}=1 z_{i}<c_{i}\right.}\right)^{\prime}
$$

The log-likelihood function of BXGNH distribution can be derived as

where

$$
\begin{aligned}
& L_{(\underline{\Phi})}=\sum_{i=1}^{n} \boldsymbol{\nabla}_{i}\left[\begin{array}{l}
\ln (2 a b \theta \beta)+(\theta-1) \ln \left(\beta z_{i}-1\right)+(2 b-1) \ln \varpi_{i} \\
-s_{i}-3 b \ln \varpi_{i}-\left(\frac{\varpi_{i}^{b}}{1-\varpi_{i}^{b}}\right)^{2}+(a-1) \ln \left(1-\boldsymbol{O}_{Q_{i}}\right)
\end{array}\right] \\
& -\sum_{i=1}^{n}\left(1-\nabla_{i}\right) \ln \left[1-\left(1-\boldsymbol{O}_{Q_{i}}\right)^{a}\right],
\end{aligned}
$$

$$
\varpi_{i}=1-\boldsymbol{\mathcal { O }}_{\left[z_{i} ; \theta, \beta\right]}, S_{i}=1-\left(z_{i} \beta+1\right)^{\theta}, \boldsymbol{\mathcal { O }}_{\mathcal{Q}_{i}}=\exp \left(-\boldsymbol{Q}_{i}^{2}\right), \boldsymbol{Q}_{i}=\frac{\varpi_{i}^{b}}{1-\varpi_{i}^{b}} .
$$

The score functions are obtained as follows

$$
\begin{aligned}
& \frac{\partial L_{(\underline{\Phi})}}{\partial a}=\sum_{i=1}^{n} \boldsymbol{\nabla}_{i}\left[\frac{1}{a}+\ln \left(1-\boldsymbol{\mathcal { O }}_{Q_{i}}\right)\right]-\sum_{i=1}^{n}\left(1-\boldsymbol{\nabla}_{i}\right) \frac{\left(1-\boldsymbol{\mathcal { O }}_{Q_{i}}\right)^{a} \ln \left(1-\boldsymbol{\mathcal { O }}_{Q_{i}}\right)}{1-\left(1-\boldsymbol{\mathcal { O }}_{\mathcal{Q}_{i}}\right)^{a}} \\
& \frac{\partial L_{(\underline{\Phi})}}{\partial b}=\sum_{i=1}^{n} \nabla_{i}\left[\frac{1}{b}-\ln \left(\varpi_{i}\right)-\frac{2 \varpi_{i}^{2 b} \ln \left(\varpi_{i}\right)}{\left(1-\varpi_{i}^{b}\right)^{3}}+\frac{2(a-1) \varpi_{i}^{2 b} \ln \left(\varpi_{i}\right) \boldsymbol{\mathcal { O }}_{Q_{i}}}{\left(1-\varpi_{i}^{b}\right)^{3}\left(1-\boldsymbol{\mathcal { O }}_{Q_{i}}\right)}\right] \\
& -2 a \sum_{i=1}^{n} \frac{\left(1-\boldsymbol{\nabla}_{i}\right) \varpi_{i}^{2 b} \ln \left(\varpi_{i}\right) \boldsymbol{\mathcal { O }}_{Q_{i}}\left(1-\boldsymbol{\mathcal { O }}_{Q_{i}}\right)^{a-1}}{\left(1-\varpi_{i}^{b}\right)^{3}\left[1-\left(1-\boldsymbol{\mathcal { O }}_{\mathcal{Q}_{i}}\right)^{a}\right]}, \\
& \frac{\partial L_{(\underline{\boldsymbol{\Phi}})}}{\partial \theta} \\
& =\sum_{i=1}^{n} \boldsymbol{\nabla}_{i}\left[\begin{array}{c}
\frac{1}{\theta}+\left(1+\left(1+\beta z_{i}\right)^{\theta}\right) \ln \left(1+\beta z_{i}\right)-\frac{(b-1)\left(\beta z_{i}+1\right)^{\theta} \ln \left(1+\beta z_{i}\right) \exp \left(-s_{i}\right)}{\varpi_{i}} \\
-\frac{2 b\left(\beta z_{i}+1\right)^{\theta} \ln \left(1+\beta z_{i}\right) \exp \left(-s_{i}\right) \boldsymbol{Q}_{i}^{2}}{\varpi_{i}\left(1-\varpi_{i}^{b}\right)}+\frac{2(a-1) b\left(\beta z_{i}+1\right)^{\theta} \ln \left(1+\beta z_{i}\right) \exp \left(-s_{i}\right) \boldsymbol{Q}_{i}^{2} \boldsymbol{O}_{Q_{i}}}{\varpi_{i}\left(1-\varpi_{i}^{b}\right)\left(1-\boldsymbol{O}_{Q_{i}}\right)}
\end{array}\right] \\
& -2 a b \sum_{i=1}^{n} \frac{\left(1-\boldsymbol{\nabla}_{i}\right)\left(\beta z_{i}+1\right)^{\theta} \ln \left(1+\beta z_{i}\right) \exp \left(-s_{i}\right) \boldsymbol{Q}_{i}^{2} \boldsymbol{\mathcal { O }}_{\boldsymbol{Q}_{i}}\left(1-\boldsymbol{\mathcal { O }}_{\boldsymbol{Q}_{i}}\right)^{a-1}}{\varpi_{i}\left(1-\varpi_{i}^{b}\right)\left[1-\left(1-\boldsymbol{\mathcal { O }}_{\mathcal{Q}_{i}}\right)^{a}\right]},
\end{aligned}
$$

and

$$
\frac{\partial L_{(\underline{\Phi})}}{\partial \beta}=\sum_{i=1}^{n} \nabla_{i}\left[\begin{array}{c}
\frac{1}{\beta}+\frac{(\theta-1) z_{i}}{1+\beta z_{i}}+\theta z_{i}\left(1+\beta z_{i}\right)^{\theta-1}+\frac{(5 b-1) \theta z_{i} \exp \left(-s_{i}\right)\left(\beta z_{i}+1\right)^{\theta-1}}{\varpi_{i}} \\
+\frac{2 b \theta z_{i} \exp \left(-s_{i}\right) \boldsymbol{Q}_{i}^{2}\left(\beta z_{i}+1\right)^{\theta-1}}{\varpi_{i}\left(1-\varpi_{i}^{b}\right)}-\frac{2(a-1) b \theta z_{i} \exp \left(-s_{i}\right) \boldsymbol{Q}_{i}^{2} \boldsymbol{\mathcal { O }}_{\mathcal{Q}_{i}}\left(\beta z_{i}+1\right)^{\theta-1}}{\varpi_{i}\left(1-\varpi_{i}^{b}\right)\left(1-\boldsymbol{O}_{\boldsymbol{Q}_{i}}\right)}
\end{array}\right]
$$




$$
-2 a \theta b \sum_{i=1}^{n} \frac{\left(1-\boldsymbol{\nabla}_{i}\right) z_{i} \exp \left(-s_{i}\right) \boldsymbol{Q}_{i}^{2} \boldsymbol{\mathcal { O }}_{\mathcal{Q}_{i}}\left(\beta z_{i}+1\right)^{\theta-1}\left(1-\boldsymbol{\mathcal { O }}_{Q_{i}}\right)^{a-1}}{\varpi_{i}\left(1-\varpi_{i}^{b}\right)\left[1-\left(1-\boldsymbol{\mathcal { O }}_{\mathcal{Q}_{i}}\right)^{a}\right]} .
$$

Maximum likelihood estimators of the unknown parameters can be obtained using various techniques, either software R, EM algorithm or Newton Raphson method.

\section{Modified chi-square type test for right censored data}

In this work, we are interested by the modified chi-square type test proposed in Bagdonavicius and Nikulin (2011), Bagdonavicius et al., (2013), for parametric models with right censored data. Based on maximum likelihood estimators on non-grouped data, this statistic test is also based on the differences between the numbers of observed failures and the numbers of expected failures in the grouped intervals chosen. For this, random grouping intervals are considered as data functions. The description of the construction of this chi-square type test is developed in Voinov et al. (2013). The statistic test is defined as follows. Suppose that $Z_{1}, Z_{2}, \ldots \ldots, Z_{n}$ is a random sample with right censoring from a parametric model, and a finite time $\tau$. The statistic test is defined as

$$
Y_{n}^{2}=\sum_{j=1}^{n} \frac{1}{U_{j}}\left(U_{j}-e_{j}\right)^{2}+Q
$$

where $U_{j}$ and $e_{j}$ are the observed and the expected numbers of failure in grouping intervals, and $Q$ is given in Abouelmagd et al., (2019a,b), Mansour et al., (2020a,b), Yadav et al., (2020), Salah et al., (2020), Goual and Yousof (2020), Goual et al., (2020), Ibrahim et al., (2020 and 2021a). The limits $\rho_{j}$ of $k$ random grouping intervals $I_{j}=$ $\left[\rho_{j-1}, \rho_{j}[\right.$ are chosen such as the expected failure times to fall into these intervals are the same for each $j=1, \ldots, k-$ 1 . The estimated $\hat{\rho}_{j}$ is defined by

$$
\hat{\rho}_{j}=\mathrm{H}^{-1}\left[\frac{\boldsymbol{E}_{j}-\sum_{j=1}^{i-1} H_{\underline{\Phi}}\left(z_{\ell}\right)}{n-i+1}, \underline{\widehat{\Phi}}\right], j=1, \ldots, k
$$

where $H_{\underline{\Phi}}\left(z_{\ell}\right)$ is the cumulative hazard function (CHRF) of our new distribution. This statistic test $Y_{n}^{2}$ follows a chisquare distribution. Suppose that $Z_{1}, Z_{2}, \ldots \ldots, Z_{n}$ is a random sample with right censoring from BXGNH distribution and a finite time $\tau$. In our case, the estimated $\hat{\rho}_{j}$ is obtained where $H_{\underline{\Phi}}\left(z_{\ell}\right)$ is the cumulative hazard function (CHRF) of the BXGNH distribution. To calculate the quadratic form $Q$ of the statistic $Y_{n}^{2}$, and as its distribution does not depend on the parameters, so we can use the estimated matrices $\widehat{W}, \hat{C}$ and the estimated information matrix $\hat{I}$. Therefore, the estimated matrix $\widehat{W}$ can be deducted from $\hat{C}$. We need also the information matrix $\hat{I}$ of the BXGNH distribution with right censoring. After difficult calculations and some simplifications, we have obtained the elements of the matrix can be easily derived. As all the components of the statistic are given explicitly, then we obtain the statistic test for the BXGNH distribution with unknown parameters and right censored data. This statistic follows a chi-square distribution with $k$ degrees of freedom.

\section{Simulated censored samples}

In this section we conduct an important simulation study to consolidate our results. For this, $N=10,000$ censored samples (with sizes: $n=25,50,130,350,500,1000$ ) from BXGNH distribution are simulated. We generate the simulated samples with various parameters. Using R software and BB algorithm (see Ravi and Gilbert (2009)), means simulated maximum likelihood estimators (MSMLEs) and their mean square errors (MSEs) are calculated and given in Tables 8, 9, 10 and 11. As shown in these results, MSEs are convergent.

Table 8: MSMLEs $\mathrm{a}=0.5, \mathrm{~b}=1.2, \beta=0.1, \theta=0,75$ and theirs MSEs.

\begin{tabular}{ccccc}
\hline $\mathrm{N}=10.000$ & $\mathrm{a}=0.5$ & $\mathrm{~b}=1.2$ & $\theta=0.1$ & $\beta=0.75$ \\
\hline $\mathrm{n}_{1}=25$ & $0.5197(0.0069)$ & $1.1703(0.0073)$ & $0.1097(0.0052)$ & $0.7527(0.0042)$ \\
$\mathrm{n}_{2}=50$ & $0.5146(0.0057)$ & $1.1786(0.0062)$ & $0.1056(0.0043)$ & $0.7522(0.0037)$ \\
$\mathrm{n}_{3}=130$ & $0.5126(0.0048)$ & $1.1816(0.0056)$ & $0.1023(0.0035)$ & $0.7518(0.0031)$ \\
$\mathrm{n}_{4}=350$ & $0.5092(0.0036)$ & $1.1883(0.0043)$ & $0.1015(0.0021)$ & $0.7512(0.0025)$ \\
$\mathrm{n}_{5}=500$ & $0.5076(0.0025)$ & $1.1924(0.0032)$ & $0.1008(0.0013)$ & $0.7509(0.0017)$ \\
$\mathrm{n}_{6}=1000$ & $0.5010(0.0018)$ & $1.1993(0.0021)$ & $0.1002(0.0005)$ & $0.7503(0.0006)$ \\
\hline
\end{tabular}


Table 9: MSMLEs $a=4, b=2, \beta=0.25, \theta=5$ and theirs MSEs.

\begin{tabular}{ccccc}
\hline $\mathrm{N}=10.000$ & $\mathrm{a}=4$ & $\mathrm{~b}=2$ & $\theta=2.5$ & $\beta=5$ \\
\hline $\mathrm{n}_{1}=25$ & $3.9715(0.0092)$ & $2.0192(0.0086)$ & $2.5246(0.0068)$ & $4.9618(0.0078)$ \\
$\mathrm{n}_{2}=50$ & $3.9777(0.0058)$ & $2.0177(0.0072)$ & $2.5202(0.0057)$ & $4.9736(0.0069)$ \\
$\mathrm{n}_{3}=130$ & $3.9804(0.0044)$ & $2.0156(0.0063)$ & $2.5197(0.0049)$ & $4.9782(0.0058)$ \\
$\mathrm{n}_{4}=350$ & $3.9873(0.0038)$ & $2.0124(0.0052)$ & $2.5136(0.0039)$ & $4.9824(0.0044)$ \\
$\mathrm{n}_{5}=500$ & $3.9898(0.0027)$ & $2.0091(0.0040)$ & $2.5089(0.0030)$ & $4.9873(0.0037)$ \\
$\mathrm{n}_{6}=1000$ & $3.9937(0.0012)$ & $2.0026(0.0033)$ & $2.5016(0.0022)$ & $4.9912(0.0028)$ \\
\hline
\end{tabular}

Table 10: MSMLEs $a=2, b=1.5, \theta=2, \beta=0.9$ and theirs MSEs.

\begin{tabular}{ccccc}
\hline $\mathrm{N}=10.000$ & $\mathrm{a}=2$ & $\mathrm{~b}=1.5$ & $\theta=2$ & $\beta=0.9$ \\
\hline $\mathrm{n}_{1}=25$ & $1.9468(0.0123)$ & $1.5176(0.0096)$ & $2.0133(0.0092)$ & $0.9102(0.0073)$ \\
$\mathrm{n}_{2}=50$ & $1.9557(0.0108)$ & $1.5143(0.0087)$ & $2.0072(0.0081)$ & $0.9078(0.0053)$ \\
$\mathrm{n}_{3}=130$ & $1.9668(0.0091)$ & $1.5126(0.0074)$ & $2.0052(0.0076)$ & $0.9043(0.0042)$ \\
$\mathrm{n}_{4}=350$ & $1.9787(0.0082)$ & $1.5102(0.0067)$ & $2.0038(0.0051)$ & $0.9022(0.0035)$ \\
$\mathrm{n}_{5}=500$ & $1.9816(0.0064)$ & $1.5083(0.0055)$ & $2.0026(0.0037)$ & $0.9017(0.0024)$ \\
$\mathrm{n}_{6}=1000$ & $1.9943(0.0056)$ & $1.5031(0.0042)$ & $2.0013(0.0019)$ & $0.9008(0.0010)$ \\
\hline
\end{tabular}

Table 11: MSMLEs $a=0.8, b=1, \theta=0.05, \beta=0.35$ and theirs MSEs.

\begin{tabular}{ccccc}
\hline $\mathrm{N}=10.000$ & $\mathrm{a}=0.8$ & $\mathrm{~b}=1$ & $\theta=0.05$ & $\beta=0.35$ \\
\hline $\mathrm{n}_{1}=25$ & $0.8208(0.0091)$ & $0.9516(0.0112)$ & $0.0519(0.0038)$ & $0.3446(0.0063)$ \\
$\mathrm{n}_{2}=50$ & $0.8196(0.0071)$ & $0.9696(0.0096)$ & $0.0516(0.0033)$ & $0.3472(0.0048)$ \\
$\mathrm{n}_{3}=130$ & $0.8135(0.0052)$ & $0.9754(0.0088)$ & $0.0513(0.0028)$ & $0.3479(0.0034)$ \\
$\mathrm{n}_{4}=350$ & $0.8112(0.0036)$ & $0.9836(0.0075)$ & $0.0509(0.0023)$ & $0.3483(0.0027)$ \\
$\mathrm{n}_{5}=500$ & $0.8086(0.0026)$ & $0.9873(0.0063)$ & $0.0503(0.0012)$ & $0.3491(0.0020)$ \\
$\mathrm{n}_{6}=1000$ & $0.8013(0.0018)$ & $0.9926(0.0052)$ & $0.0501(0.0004)$ & $0.3497(0.0011)$ \\
\hline
\end{tabular}

\section{Simulation results for test statistic $Y^{2}$}

In order to study the performance of the test statistic proposed in this work, a simulation study has been carried out. Thus, for testing the null hypothesis $H_{0}$ with respect to sample belongs to BXGNH distribution, we draw 10,000 samples data from BXGNH model with different sample sizes and different parameter values to calculate $Y^{2}$ statistic. Then, we compute the number of cases of rejection of the null hypothesis $H_{0}$, when the values of criteria statistic $Y^{2}$, calculated as shown above, are superior to $\chi_{\theta}^{2}(k)$ (the quantile of the chi-square distribution with $k$ degrees of freedom).

Table 12: Simulated levels of significance for modified test against their theoretical values $10 \%, 5 \%, 2 \%$

\begin{tabular}{cccccc}
\hline \multicolumn{5}{c}{ test against their theoretical values } & $10,5 \%, 2 \%$ \\
\hline$\alpha=10 \%$ & 0.1012 & 0.0993 & 0.0982 & 0.0976 & 0.0963 \\
$\alpha=05 \%$ & 0.0490 & 0.0481 & 0.0472 & 0.0463 & 0.0459 \\
$\alpha=02 \%$ & 0.0216 & 0.0198 & 0.0189 & 0.0172 & 0.0163 \\
& & & & & \\
& $\mathrm{n}_{5}=1000$ & $\mathrm{n}_{4}=500$ & $\mathrm{n}_{3}=350$ & $\mathrm{n}_{2}=150$ & $\mathrm{n}_{1}=50$ \\
$\alpha=10 \%$ & 0.1063 & 0.1034 & 0.1002 & 0.0991 & 0.0983 \\
$\alpha=05 \%$ & 0.482 & 0.473 & 0.0462 & 0.0444 & 0.0428 \\
$\alpha=02 \%$ & 0.0176 & 0.0157 & 0.0143 & 0.0124 & 0.0113 \\
& & & & & \\
$\alpha=10 \%$ & 0.1008 & 0.0996 & 0.0987 & 0.0976 & 0.0966 \\
$\alpha=05 \%$ & 0.0502 & 0.0494 & 0.0476 & 0.0443 & 0.0426 \\
$\alpha=02 \%$ & 0.0201 & 0.0194 & 0.0186 & 0.0173 & 0.0162 \\
& & & & & \\
$\alpha=10 \%$ & 0.0976 & 0.0965 & 0.0954 & 0.0948 & 0.0942 \\
$\alpha=05 \%$ & 0.0466 & 0.0454 & 0.0448 & 0.0442 & 0.0434 \\
$\alpha=02 \%$ & 0.0198 & 0.0192 & 0.0184 & 0.0173 & 0.0162 \\
\hline
\end{tabular}


We give a comparison between the different theoretical values of significance level $\theta$ (with $\theta=0.10, \theta=0.05, \theta=$ 0.02 ) and their simulated levels (empirical levels) of significance in Table 12. As can be seen, the values of the calculated empirical levels of $Y^{2}$ test are very close to those of their corresponding theoretical levels of the chi-square distributions with $k$ degrees of freedom. Thus, we conclude that the proposed test is well suited to the BXGNH distribution.

\section{Four censored applications for validation}

The modified Bagdonavicius-Nikulin test for validation under the right censored data is applied for four real and right censored data sets.

\section{Application 1:}

The first data set, we apply the results obtained from this study to real data established from reliability (see Crowder et al., (1991)). In an experiment to obtain information on the strength of a certain type of braided cord after the weather, the forces of 48 pieces of cord having resisted for a determined time were studied. The right censored force values observed are given as $26.8^{*}, 29.6^{*}, 33.4^{*}, 35^{*}, 36.3,40^{*}, 41.7,41.9^{*}, 42.5^{*}, 43.9,49.9,50.1,50.8,51.9,52.1,52.3$, 52.3, 52.4, 52.6, 52.7, 53.1, 53.6, 53.6, 53.9, 53.9, 54.1, 54.6, 54.8, 54.8, 55.1, 55.4, 55.9, 56, 56.1, 56.5, 56.9, 57.1, $57.1,57.3,57.7,57.8,58.1,58.9,59,59.1,59.6,60.4,60.7$.

\section{Application 2:}

The second data (see John et al., (1997)) set, In a study designed to determine if female breast cancer patients, originally classified as lymph node negative by standard light microscopy (SLM), could be more accurately classified by immunohistochemical (IH) examination of their lymph nodes with an anti-cytokeratin monoclonal antibody cocktail, identical sections of lymph nodes were sequentially examined by SLM and IH. The significance of this study is that $16 \%$ of patients with negative axillary lymph nodes, by standard pathological examination, develop recurrent disease within 10 years. Forty-five female breast-cancer patients with negative axillary lymph nodes and a minimum 10-year follow-up were selected from The Ohio State University Hospitals Cancer Registry. Of the 45 patients, 9 were immunoperoxidase positive, and the remaining 36 remained negative. The data required for this study is shown as: $19,25,30,34,37,46,47,51,56,57,61,66,67,74,78,86,122^{*}, 123^{*}, 130^{*}, 130^{*}, 133^{*}, 134^{*}, 136^{*}, 141^{*}$, $143^{*}, 148^{*}, 151^{*}, 152^{*}, 153^{*}, 154^{*}, 156^{*}, 162^{*}, 164^{*}, 165^{*}, 182^{*}, 189^{*}$.

\section{Application 3:}

The third data set (see John et al., (1997)) has reported survival data on 26 psychiatric inpatients admitted to the university of Iowa hospitals during the years 1935-1948. This sample is part of a larger study of psychiatric Inpatients discussed by Tsuang and Woolson (1977). Data for each patient consists of age at rest admission to the hospital, sex, number of years of follow-up (years from admission to death or censoring) and patient status at the follow-up time. The data is given as $1,1,2,11,14,22,22,24,25,26,28,30^{*}, 30^{*}, 31^{*}, 31^{*}, 32,33^{*}, 33^{*}, 34^{*}, 35,35^{*}, 35^{*}, 36^{*}$, $37^{*}, 39^{*}, 40$. (* indicates the censorship).

\section{Application 4:}

The forth data set (see John et al., (1997)) consists sample data from 50 patients with acute myeloid leukemia, reported to the International Register of Bone Marrow Transplants. These patients had an allogeneic bone marrow transplant where the Histocompatibility Leukocyte Antigen (HLA) homolog marrow was used to rebuild their immune systems. The data required for this study is shown as $0.030,0.493,0.855,1.184,1.283,1.480,1.776,2.138,2.5,2.763,2.993$, $3.224,3.421,4.178,4.441^{*}, 5.691,5.855^{*}, 6.941^{*}, 6.941,7.993^{*}, 8.882,8.882,9.145^{*}, 11.480,11.513,12.105^{*}$, $12.796,12.993^{*}, 13.849^{*}, 16.612^{*}, 17.138^{*}, 20.066,20.329^{*}, 22.368^{*}, 26.776^{*}, 28.717^{*}, 28.717 *, 32.928^{*}, 33.783^{*}$ , 34.211*, 34.770*, 39.539*, 41.118*, 45.033*, 46.053*, 46.941*, 48.289*, 57.401*, 58.322*, 60.625*. All results are for the four real data sets listed in Tables 13 and Table 14.

Table 13: The values of $\mathrm{Y}^{2}$ for the four data sets.

\begin{tabular}{cccccc}
\hline & $\mathrm{a}$ & $\mathrm{b}$ & $\theta$ & $\beta$ & $\mathrm{Y}^{2}$ \\
\hline Data 1 & 4.1632 & 1.3646 & 2.3939 & 3.5626 & $9.6768<\chi_{5}{ }^{2}=11.0705$ \\
\hline Data 2 & 1.8468 & 0.8196 & 1.5284 & 2.3467 & $7.1293<\chi_{4}{ }^{2}=9.48770$ \\
\hline Data 3 & 1.3167 & 2.6142 & 0.9868 & 1.0367 & $7.9634<\chi_{4}{ }^{2}=9.48770$ \\
Data 4 & 2.0689 & 0.9109 & 1.0410 & 0.9417 & $8.1464<\chi_{5}{ }^{2}=11.0705$ \\
\hline
\end{tabular}


Table 14: $\mathrm{Y}^{2}$ values for all competing models.

\begin{tabular}{ccccc}
\hline Distribution & Data 1 & Data 2 & Data 3 & Data 4 \\
\cline { 2 - 5 } & $\mathrm{Y}_{1}^{2}$ & $\mathrm{Y}_{2}^{2}$ & $\mathrm{Y}_{3}^{2}$ & $\mathrm{Y}_{4}^{2}$ \\
\hline BXGNH & $\mathbf{9 . 6 7 6 8}$ & $\mathbf{7 . 1 2 9 3}$ & $\mathbf{7 . 9 6 3 4}$ & $\mathbf{8 . 1 7 6 4}$ \\
RGNH & 9.8226 & 7.2617 & 8.1245 & 8.2018 \\
RNH & 9.6907 & 8.1676 & 8.3346 & 8.3476 \\
NH & 9.7617 & 7.3406 & 8.2642 & 8.2106 \\
GaNH & 10.1173 & 7.4118 & 8.4678 & 8.5683 \\
OLNH & 9.7304 & 7.6213 & 8.0331 & 8.2189 \\
PRHRNH & 9.8306 & 7.7361 & 8.5349 & 8.8607 \\
GNH & 9.9163 & 7.8273 & 8.1736 & 8.2267 \\
BNH & 9.9907 & 8.2361 & 8.9664 & 8.7776 \\
\hline
\end{tabular}

If we consider significance level $\theta=0.05$, the critical value $\chi_{5}^{2}=11.0705$, and $\chi_{4}^{2}=9,4877$ according to the results obtained in Table 10 and 11, these data sets can be fitted by several distributions but the lowest value of the goodness-of-fit statistic is $Y^{2}=7.1293$ corresponding to the proposed model BXGNH.

\section{Conclusions}

In this work, a new three-parameter extension of the generalized Nadarajah-Haghighi model (GNH) called Burr X GNH (BXGNH) model is introduced and studied. Some of its statistical properties have been derived. Characterization results based on a simple relationship between two truncated moments, in terms of the HRF and based on the conditional expectation of a function of the RV are presented with details. The new PDF can be expressed as a linear mixture of the generalized Nadarajah-Haghighi PDF. It was shown that HRF of the new extension can be increasing, decreasing, bathtub (U, upside-down, upside-down-constant, increasing-constant or constant. Variance, skewness and kurtosis were studied by a numerical analysis. The skewness of the BXGNH can range in the interval $(0.86,6.96)$, whereas the skewness of the standard GNH distribution varies only in the interval $(0.435,3.17)$. Further, the spread for the BXGNH kurtosis is ranging from 4.61 to 1225.3, whereas the spread for the standard GNH kurtosis only varies only from 3.37 to 9 . A numerical and graphical Monte Carlo simulation study is conducted to assess and analyze the performance of the estimation method. The flexibility of the BXGNH model is illustrated via a real data set. Different non-Bayesian estimation methods under uncensored scheme are considered such as the maximum likelihood estimation method, Anderson Darling estimation method, ordinary least square estimation method, Cramér-von-Mises estimation method, weighted least square estimation method, left tail-Anderson Darling estimation method and right tail Anderson Darling estimation method. Numerical simulations Are performed for comparing the estimation methods using different sample sizes the three different combinations of parameters. The Barzilai-Borwein algorithm is employed via a simulation study for assessing the performance of the estimators with different sample sizes as sample size tends to $+\infty$. An application is presented for measuring the flexibility and the importance of the new model and also used for comparing the competitive distributions under the uncensored scheme. Using the approach of the Bagdonavicius-Nikulin chi-square goodness-of-fit test for validation under the right censored data, we propose a modified chi-square goodness-of-fit test for the BXGNH model. Based on the maximum likelihood estimators on initial data, the modified Bagdonavicius-Nikulin chi-square goodness-of-fit test recovers the loss in information. The modified Bagdonavicius-Nikulin chi-square goodness-of-fit test for validation under the right censored data is applied for four real and right censored data sets.

As a potential future work, we could use the most common copulas for deriving many bivariate extensions with application to bivariate real data sets (see Al-babtain et al., (2020), Mansour et al., (2020c,e,f), Ibrahim et al., (2020, 2021b), Elgohari and Yousof (2020a,b). Yousof et al., (2020, 2021a,b) and Ali et al., (2021a,b) for more details). Also, the Nikulin-Rao-Robson test can be employed for distributional validation of the BXGNH model (see Goual and Yousof (2020) and Goual et al., (2019 and 2020) for more details).

\section{References}

1. Aarset, M. V. (1987). How to identify bathtub hazard rate. IEEE Transactions on Reliability, 36, $106-108$.

2. Abouelmagda, T. H. M., Hameda, M. S., Hamedani, G. G., Ali, M. M., Goual, H., Korkmaze, M. C. and Yousof, H. M. (2019a). The zero truncated Poisson Burr X family of distributions with properties, characterizations, applications, and validation test. Journal of Nonlinear Sciences and Applications, 12(5), 314-336. 
3. Abouelmagd, T. H. M., Hamed, M. S., Handique, L., Goual, H., Ali, M. M., Yousof, H. M. and Korkmaz, M. C. (2019b). A new class of distributions based on the zero truncated Poisson distribution with properties and applications, Journal of Nonlinear Sciences \& Applications (JNSA), 12(3), 152-164.

4. Al-babtain, A. A., Elbatal, I. and Yousof, H. M. (2020). A new flexible three-parameter model: properties, Clayton copula, and modeling real data. Symmetry, 12(3), 440.

5. Ali, M. M., Ibrahim, M. and Yousof, H. M. (2021a). Expanding the Burr X Model: Properties, Copula, Real Data Modeling and Different Methods of Estimation. Optimal Decision Making in Operations Research \& Statistics: Methodologies and Applications, CRC Press, To appear.

6. Ali, M. M., Yousof, H. M. and Ibrahim, M. (2021b). A New Version of the Generalized Rayleigh Distribution with Copula, Properties, Applications and Different Methods of Estimation. Optimal Decision Making in Operations Research \& Statistics: Methodologies and Applications, CRC Press, To appear.

7. Alizadeh, M., Jamal, F., Yousof, H. M., Khanahmadi, M. and Hamedani, G. G. (2020). Flexible Weibull generated family of distributions: characterizations, mathematical properties and applications. University Politehnica of Bucharest Scientific Bulletin-Series A-Applied Mathematics and Physics, 82(1), 145-150.

8. Alizadeh, M., Rasekhi, M., Yousof, H. M., Ramires, T. G. and Hamedani G. G. (2018). Extended exponentiated Nadarajah-Haghighi model: mathematical properties, characterizations and applications. Studia Scientiarum Mathematicarum Hungarica, 55, 498-522.

9. Altun, E., Yousof, H. M., Chakraborty, S. and Handique, L. (2018a). Zografos-Balakrishnan Burr XII distribution: regression modeling and applications. International Journal of Mathematics and Statistics, 19(3), 46-70.

10. Altun, E., Yousof, H. M. and Hamedani, G. G. (2018b). A new log-location regression model with influence diagnostics and residual analysis. Facta Universitatis, Series: Mathematics and Informatics, 33(3), 417-449.

11. Altun, E., Yousof, H. M. and Hamedani, G. G. (2018c). A new generalization of generalized half-normal distribution: properties and regression models. Journal of Statistical Distributions and Applications, 5(1), 7.

12. Altun, E., Yousof, H. M. and Hamedani G. G. (2018d). A new flexible extension of the generalized halfnormal lifetime model with characterizations and regression modeling. Bulletin of Computational Applied Mathematics, 6(1), 83-115.

13. Altun, E., Yousof, H. M. and Hamedani G. G. (2018e). A Flexible Extension of Generalized Half-Normal Distribution: Characterizations and Regression Models. International Journal of Applied Mathematics and Statistics, 57(3), 27-49.

14. Altun, E., Yousof, H. M. and Hamedani, G. G. (2021). The Gudermannian generated family of distributions with characterizations, regression models and applications, Studia Scientiarum Mathematicarum Hungarica, forthcoming.

15. Bagdonavičius, V. and Nikulin, M. (2011). Chi-square goodness-of-fit test for right censored data, International Journal of Applied Mathematics and Statistics, 24, 30-50.

16. Bagdonavičius, V., Levuliene, R., J. and Nikulin, M., (2013). Chi-square goodness-of-fit tests for parametric accelerated failure time models, Communications in Statistics- Theory and Methods, 42(15), 2768-2785.

17. Bhatti, F. A., Hamedani, G. G., Korkmaz, M.C., Yousof, H. M. and Ahmad, M. (2020a). On the new modified Burr XII distribution: development, properties, characterizations and applications, Journal of data science, forthcoming.

18. Bhatti, F. A., Hamedani, G. G., Korkmaz, M. C., Cordeiro, G. M., Yousof, H. M. and Ahmad, M. (2019). On Burr III Marshal Olkin family: development, properties, characterizations and applications. Journal of Statistical Distributions and Applications, 6(1), 1-21.

19. Bhatti, F. A., Hamedani, G. G., Yousof, H. M., Ali, A. and Ahmad, M. (2018). On Modified Burr XII-Inverse Exponential Distribution: Properties, Characterizations and Applications. J. Biostat Biom: JBSB-106.

20. Bhatti, F. A., Hamedani, G. G., Yousof, H. M., Ali, A. and Ahmad, M. (2020b). On Modified Burr XIIInverse Weibull Distribution: Properties and Applications. Pakistan Journal of Statistics and Operation Research 16(4),721-735.

21. Bhatti, F. A., Hamedani, G. G., Yousof, H. M. and Ali, A. (2021). On the Burr III-Moment Exponential Distribution. Thailand statistician, forthcoming.

22. Choulakian, V., Stephens, M.A. (2001). Goodness-of-fit for the generalized Pareto distribution. Technometrics, 43, 478--484.

23. Crowder, M. J., Kimber A. C., Smith R. L and Sweeting, T. J. (1991). Statistical analysis of reliability data, CHAPMAN \&HALL/CRC

24. Dias, C. R. B., Alizadeh, M. and Cordeiro, G. M. (2018). The beta Nadarajah-Haghighi distribution. Hacettepe Journal of Mathematics and Statistics, 47, 13021323. 
25. Elgohari, H. and Yousof, H. M. (2020a). A Generalization of Lomax Distribution with Properties, Copula and Real Data Applications. Pakistan Journal of Statistics and Operation Research, 16(4), 697-711.

26. Elgohari, H. and Yousof, H. M. (2020b). New Extension of Weibull Distribution: Copula, Mathematical Properties and Data Modeling. Statistics, Optimization \& Information Computing, 8(4), 972-993.

27. Elsayed, H. A. H. and Yousof, H. M. (2019). The Burr X Nadarajah Haghighi distribution: statistical properties and application to the exceedances of flood peaks data. Journal of Mathematics and Statistics, 15, 146-157.

28. El-Morshedy, M., Alshammari, F. S., Hamed, Y. S., Eliwa, M. S., Yousof, H. M. (2021). A New Family of Continuous Probability Distributions. Entropy, 23, 194.

29. Gad, A. M., Hamedani, G. G., Salehabadi, S. M. and Yousof, H. M. (2019). The Burr XII-Burr XII distribution: mathematical properties and characterizations. Pakistan Journal of Statistics, 35(3), 229-248.

30. Glänzel, W., A. (1987) characterization theorem based on truncated moments and its application to some distribution families, Mathematical Statistics and Probability Theory (Bad Tatzmannsdorf, 1986), Vol. B, Reidel, Dordrecht, 75--84.

31. Glänzel, W. (1990). Some consequences of a characterization theorem based on truncated moments, Statistics: A Journal of Theoretical and Applied Statistics, 21 (4), 613-618.

32. Goual, H. and Yousof, H. M. (2020). Validation of Burr XII inverse Rayleigh model via a modified chisquared goodness-of-fit test. Journal of Applied Statistics, 47(3), 393-423.

33. Goual, H., Yousof, H. M. and Ali, M. M. (2019). Validation of the odd Lindley exponentiated exponential by a modified goodness of fit test with applications to censored and complete data. Pakistan Journal of Statistics and Operation Research, 15(3), 745-771.

34. Goual, H., Yousof, H. M. and Ali, M. M. (2020). Lomax inverse Weibull model: properties, applications, and a modified Chi-squared goodness-of-fit test for validation. Journal of Nonlinear Sciences \& Applications (JNSA), 13(6), 330-353.

35. Hamedani, G.G. (2013). On certain generalized gamma convolution distributions II, Technical Report, No. 484, MSCS, Marquette University, 2013.

36. Ibrahim, M. (2020). The generalized odd Log-logistic Nadarajah Haghighi distribution: statistical properties and different methods of estimation. Journal of Applied Probability and Statistics, 15(2), 61-84.

37. Ibrahim, M., Altun, E., Goual, H., and Yousof, H. M. (2021a). Modified goodness-of-fit type test for censored validation under a new Burr type XII distribution with different methods of estimation and regression modeling. Eurasian Bulletin of Mathematics, 3(3), 162-182.

38. Ibrahim. M., Handique, L., Chakraborty, S., Butt, N. S. and M. Yousof, H. (2021b). A New Three-parameter Xgamma Fréchet Distribution with Different Methods of Estimation and Applications. Pakistan Journal of Statistics and Operation Research, 17(1), 291-308.

39. Ibrahim, M. Sharma, V. K., Korkmaz, M.C., Ali, M. M., Chesneau, C. and Yousof, H. M. (2020). A Generalization of Lindley Distribution: Copula, Properties and Different Methods of Estimation. MATHSTATICA. VOL1, 1-26.

40. Ibrahim, M., Yadav, A. S., Yousof, H. M., Goual, H. and Hamedani, G. G. (2019). A new extension of Lindley distribution: modified validation test, characterizations and different methods of estimation. Communications for Statistical Applications and Methods, 26(5), 473-495.

41. John, P. Klein, Melvin L. Moeschberger (1997). Survival Analysis: Techniques for Censored and Truncated Data, Statistics for Biology and Health.

42. Khalil, M. G., Hamedani, G. G. and Yousof, H. M. (2019). The Burr X exponentiated Weibull model: Characterizations, mathematical properties and applications to failure and survival times data. Pakistan Journal of Statistics and Operation Research, 141-160.

43. Korkmaz, M. C., Altun, E., Alizadeh, M. and Yousof, H. M. (2019). A new flexible lifetime model with loglocation regression modeling, properties and applications. Journal of Statistics and Management Systems, 22(5), 871-891.

44. Lemonte, A. J. (2013). A new exponential-type distribution with constant, decreasing, increasing, upsidedown bathtub and bathtub-shaped failure rate function. Computational Statistics and Data Analysis, 62, 149170.

45. Lemonte, A. J., Cordeiro, G. M. and Moreno--Arenas, G. (2016). A new useful three-parameter extension of the exponential distribution. Statistics, 50, 312-337.

46. Mansour, M. M., Ibrahim, M., Aidi, K., Shafique Butt, N., Ali, M. M., Yousof, H. M. and Hamed, M. S. (2020a). A New Log-Logistic Lifetime Model with Mathematical Properties, Copula, Modified Goodnessof-Fit Test for Validation and Real Data Modeling. Mathematics, 8(9), 1508. 
47. Mansour, M. M., Butt, N. S., Ansari, S. I., Yousof, H. M., Ali, M. M. and Ibrahim, M. (2020b). A new exponentiated Weibull distribution's extension: copula, mathematical properties and applications. Contributions to Mathematics, 1 (2020) 57-66. DOI: 10.47443/cm.2020.0018

48. Mansour, M., Korkmaz, M. Ç., Ali, M. M., Yousof, H. M., Ansari, S. I. and Ibrahim, M. (2020c). A generalization of the exponentiated Weibull model with properties, Copula and application. Eurasian Bulletin of Mathematics, 3(2), 84-102.

49. Mansour, M., Rasekhi, M., Ibrahim, M., Aidi, K., Yousof, H. M. and Elrazik, E. A. (2020d). A New Parametric Life Distribution with Modified Bagdonavičius-Nikulin Goodness-of-Fit Test for Censored Validation, Properties, Applications, and Different Estimation Methods. Entropy, 22(5), 592.

50. Mansour, M., Yousof, H. M., Shehata, W. A. and Ibrahim, M. (2020e). A new two parameter Burr XII distribution: properties, copula, different estimation methods and modeling acute bone cancer data. Journal of Nonlinear Science and Applications, 13(5), 223-238.

51. Mansour, M. M., Butt, N. S., Yousof, H. M., Ansari, S. I. and Ibrahim, M. (2020f). A Generalization of Reciprocal Exponential Model: Clayton Copula, Statistical Properties and Modeling Skewed and Symmetric Real Data Sets. Pakistan Journal of Statistics and Operation Research, 16(2), 373-386.

52. Merovci, F., Alizadeh, M., Yousof, H. M. and Hamedani, G. G. (2017). The exponentiated transmuted-G family of distributions: theory and applications. Communications in Statistics-Theory and Methods, 46(21), 10800-10822.

53. Merovci, F., Yousof, H. M. and Hamedani, G. G. (2020). The Poisson Topp Leone Generator of Distributions for Lifetime Data: Theory, Characterizations and Applications. Pakistan Journal of Statistics and Operation Research, 16(2), 343-355.

54. Nadarajah, S.and Haghighi, F. (2011). An extension of the exponential distribution. Statistics, 45, 543--558.

55. Ortega, E. M., Lemonte, A. J., Silva, G. O. and Cordeiro, G. M. (2015). New flexible models generated by gamma random variables for lifetime modeling. Journal of Applied Statistics, 42(10), 2159-2179.

56. Nascimento, A. D., Silva, K. F., Cordeiro, G. M., Alizadeh, M., Yousof, H. M. and Hamedani, G. G. (2019). The odd Nadarajah-Haghighi family of distributions: properties and applications. Studia Scientiarum Mathematicarum Hungarica, 56(2), 185-210.

57. Ravi, V. and Gilbert, P. D. (2009). BB: An R package for solving a large system of nonlinear equations and for optimizing a high-dimensional nonlinear objective function, J. Statist. Software, 32(4)

58. Salah, M. M., El-Morshedy, M., Eliwa, M. S. and Yousof, H. M. (2020). Expanded Fréchet Model: Mathematical Properties, Copula, Different Estimation Methods, Applications and Validation Testing. Mathematics, 8(11), 1949.

59. Yadav, A. S., Altun, E. and Yousof, H. M. (2019). Burr-Hatke Exponential Distribution: A Decreasing Failure Rate Model, Statistical Inference and Applications. Annals of Data Science, 1-20.

60. Yadav, A. S., Goual, H., Alotaibi, R. M., Ali, M. M. and Yousof, H. M. (2020). Validation of the ToppLeone-Lomax model via a modified Nikulin-Rao-Robson goodness-of-fit test with different methods of estimation. Symmetry, 12(1), 57.

61. Yousof, H. M., Afify, A. Z., Hamedani, G. G. and Aryal, G. (2017). The Burr X generator of distributions for lifetime data. Journal of Statistical Theory and Applications, 16, 288-305.

62. Yousof, H. M., Ali, M. M., Goual, H. and Ibrahim. M. (2021a). A new reciprocal Rayleigh extension: properties, copulas, different methods of estimation and modified right censored test for validation, STATISTICS IN TRANSITION new series, forthcoming.

63. Yousof, H. M. and Korkmaz, M. C. (2017). Topp-Leone Nadarajah-Haghighi distribution: mathematical properties and applications, International Journal of Applied Mathematics. Journal of Statisticians: Statistics and Actuarial Sciences, 2, 119-128.

64. Yousof, H. M., Chesneau, C., Hamedani, G. G. and Ibrahim. M. (2021b). The Lindley-Lindley distribution: characterizations, copula, properties, Bayesian and non-Bayesian estimation. International Journal of Mathematical Modelling \& Computations, forthcoming.

65. Yousof, H. M., Hamedani, G. G. and Ibrahim, M. (2020). The two-parameter xgamma Fréchet distribution: characterizations, copulas, mathematical properties and different classical estimation methods. Contributions to Mathematics, 2 (2020), 32-41.

66. Yousof, H. M., Korkmaz, M. C. Hamedani G. G. (2017). The odd Lindley Nadarajah- Haghighi distribution. J. Math. Comput. Sci., 7, 864-882. 